\title{
Vector-like quark interpretation for the CKM unitarity violation, excess in Higgs signal strength, and bottom quark forward-backward asymmetry
}

\author{
Kingman Cheung, ${ }^{a, b, c, d}$ Wai-Yee Keung, ${ }^{e}$ Chih-Ting Lu ${ }^{f}$ and Po-Yan Tseng ${ }^{g}$ \\ ${ }^{a}$ Physics Division, National Center for Theoretical Sciences, \\ Hsinchu, Taiwan \\ ${ }^{b}$ Department of Physics, National Tsing Hua University, \\ Hsinchu 300, Taiwan \\ ${ }^{c}$ Division of Quantum Phases and Devices, School of Physics, Konkuk University, \\ Seoul 143-701, Republic of Korea \\ ${ }^{d}$ Department of Physics, National Central University, \\ Chungli, Taiwan \\ ${ }^{e}$ Department of Physics, University of Illinois at Chicago, \\ Illinois 6060\%, U.S.A. \\ ${ }^{f}$ School of Physics, KIAS, \\ Seoul 130-722, Republic of Korea \\ ${ }^{g}$ Department of Physics and IPAP, Yonsei University, \\ Seoul 03722, Republic of Korea \\ E-mail: cheung@phys.nthu.edu.tw, keung@uic.edu, timluyu@gmail.com, \\ tpoyan1209@gmail.com
}

ABSTRACT: Due to a recent more precise evaluation of $V_{u d}$ and $V_{u s}$, the unitarity condition of the first row in the Cabibbo-Kobayashi-Maskawa (CKM) matrix: $\left|V_{u d}\right|^{2}+\left|V_{u s}\right|^{2}+\left|V_{u b}\right|^{2}=$ $0.99798 \pm 0.00038$ now stands at a deviation more than $4 \sigma$ from unity. Furthermore, a mild excess in the overall Higgs signal strength appears at about $2 \sigma$ above the standard model (SM) prediction, as well as the long-lasting discrepancy in the forward-backward asymmetry $\mathcal{A}_{\mathrm{FB}}^{b}$ in $Z \rightarrow b \bar{b}$ at LEP. Motivated from the above three anomalies we investigate an extension of the SM with vector-like quarks (VLQs) associated with the down-quark sector, with the goal of alleviating the tension among these datasets. We perform global fits of the model under the constraints coming from the unitarity condition of the first row of the CKM matrix, the $Z$-pole observables $\mathcal{A}_{\mathrm{FB}}^{b}, R_{b}$ and $\Gamma_{\text {had }}$, Electro-Weak precision observables $\Delta S$ and $\Delta T, B$-meson observables $B_{d^{-}}^{0} \bar{B}_{d}^{0}$ mixing, $B^{+} \rightarrow \pi^{+} \ell^{+} \ell^{-}$and $B^{0} \rightarrow \mu^{+} \mu^{-}$, and direct searches for VLQs at the Large Hadron Collider (LHC). Our results suggest that adding VLQs to the SM provides better agreement than the SM.

Keywords: Beyond Standard Model, Heavy Quark Physics, Higgs Physics, Quark Masses and SM Parameters

ARXIV EPRINT: 2001.02853 


\section{Contents}

1 Introduction 1

2 Standard model with extra vector-like quarks 3

2.1 Yukawa couplings and fermion masses 4

2.2 Modifications to the $W$ couplings with SM quarks 5

2.3 Modifications to the $Z$ couplings with the SM quarks 6

2.4 Minimal models $\quad 7$

3 Constraints $\quad 9$

3.1 CKM measurements 9

$3.2 Z$ boson measurements $\quad 9$

$3.3 \quad 125 \mathrm{GeV}$ Higgs precision measurements 11

3.4 Electro-Weak Precision Observables(EWPOs) 11

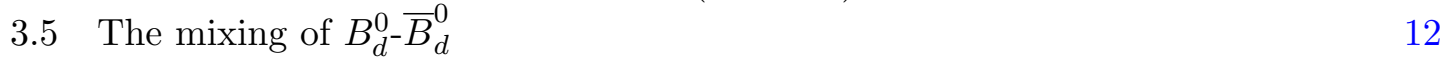

3.6 The $B^{+} \rightarrow \pi^{+} \ell^{+} \ell^{-}-14$

$\begin{array}{lll}3.7 & \text { The } B^{0} \rightarrow \mu^{+} \mu^{-} & 14\end{array}$

$\begin{array}{lll}\text { 3.8 } & \text { Direct searches for the vector-like bottom quarks } & 15\end{array}$

$\begin{array}{lll}4 & \text { Fitting } & 15\end{array}$

$\begin{array}{lll}5 & \text { Discussion } & 21\end{array}$

$\begin{array}{ll}\text { A Parameterization of the full } V_{\mathrm{CKM}}^{5 \times 5} \text { matrix } & 23\end{array}$

\section{Introduction}

The Standard Model (SM) particle content includes three families of fermions under the identical representation of the gauge symmetries $\mathrm{SU}(3)_{c} \times \mathrm{SU}(2)_{L} \times \mathrm{U}(1)_{Y}$. Each fermion family includes a quark sector (up-type and down-type quarks) and a lepton sector (charged leptons and a neutrino). The well-known quark mixing in crossing between the families is an indispensable ingredient in flavor physics. One can rotate the interaction eigenbasis to the mass eigenbasis in the quark sector through a unitary transformation, and it generates nonzero flavor mixings across the families in the charged-current interactions with the $W$ boson. The quark mixing for the three generations in the SM can be generally parameterized by the $3 \times 3$ Cabibbo-Kobayashi-Maskawa (CKM) matrix $\mathbf{V}_{\mathbf{C K M}}^{\mathbf{S M}}[1,2]$. Since $\mathbf{V}_{\mathbf{C K M}}^{\mathbf{S M}}$ is composed of two unitary matrices, unitarity of the CKM matrix shall be maintained. The existence of additional quarks beyond the three SM families shall extend the CKM matrix to a larger dimension. In such a case, the unitarity of original 3 by 3 submatrix will no longer hold. 
The recent updated measurements and analyses of $V_{u d}$ and $V_{u s}$ are briefly outlined as follows. The most precise determination of $\left|V_{u d}\right|$ is extracted from the superallowed $0^{+}-0^{+}$ nuclear $\beta$ decay measurements $[3,4]$

$$
\left|V_{u d}\right|^{2}=\frac{0.97147(20)}{1+\Delta_{R}^{V}},
$$

where $\Delta_{R}^{V}$ accounts for short-distance radiative correction. Recently, according to the dispersion relation study with experimental data of neutrino-proton scattering, the inner radiative correction with reduced hadronic uncertainties $\Delta_{R}^{V}=0.02467(22)$ was reported in ref. [5]. It significantly modified the value of $\left|V_{u d}\right|=0.97370(14)$ [4]. On the other hand, one can use various kaon decay channels to independently extract the values of $\left|V_{u s}\right|$ and $\left|V_{u s} / V_{u d}\right|$. Based on the analysis of semileptonic Kl3 decays [8] and the comparison between the kaon and pion inclusive radiative decay rates $K \rightarrow \mu \nu(\gamma)$ and $\pi \rightarrow \mu \nu(\gamma)$ [9], the values of $\left|V_{u s}\right|=0.22333(60)$ and $\left|V_{u s} / V_{u d}\right|=0.23130(50)$ are obtained in ref. [4]. As a result, the matrix-element squared of the first row of $\mathbf{V}_{\mathbf{C K M}}^{\mathbf{S M}}$

$$
\left|V_{u d}\right|^{2}+\left|V_{u s}\right|^{2}+\left|V_{u b}\right|^{2}=0.99798 \pm 0.00038
$$

which deviates from the unitarity by more than $4 \sigma[4,5] .{ }^{1}$ If this deviation is further confirmed, it may invoke additional quarks to extend the CKM matrix. ${ }^{2}$

After the final piece of the SM, Higgs boson, has been discovered in 2012 [13, 14], the precise measurements of its properties become more and more important. The SM can fully predict the signal strengths of this $125 \mathrm{GeV}$ scalar boson so that deviations from the SM predictions can help us to trace the footprint of new physics beyond the SM. Recently, the average on the Higgs-signal strengths from both ATLAS and CMS Collaborations indicated an excess at the level of $1.5 \sigma .^{3}$ If one looks more closely into each individual signal strength channel, one would find that mild $1 \sigma$ excesses appear in the majority of channels. After taking into account of all available data from the Higgs measurements, the average of the $125 \mathrm{GeV}$ Higgs signal strengths was obtained [17]

$$
\mu_{\text {Higgs }}=1.10 \pm 0.05 \text {. }
$$

One simple extension of the SM with an SU(2) doublet of vector-like quarks (VLQs) with hypercharge $-5 / 6$ can be introduced to account for the excess by reducing the bottom

\footnotetext{
${ }^{1}$ Reduction in the extracted value of $V_{u d}$ is due to the reduction of uncertainty in $\Delta_{R}^{V}$, which is made possible by a dispersion-relation based formulation of the $\gamma-W$ box contribution to the neutron and nuclear beta decays [6]. However, the value is to be taken cautiously before jumping to a conclusion, because one has to include properly the quasielastic contribution from one-nucleon knock-out as well as advanced correction from two-nucleon knock-out. On the other hand, a recent proposal to study $\Delta_{R}^{V}$ on lattice can be found in ref. [7].

${ }^{2}$ Another explanation for this deviation involves new physics in the neutrino sector with lepton-flavor universality violation [10]. Especially, they emphasized the measurements of $\left|V_{u s}\right|$ from the above kaon decays are inconsistent with the tau decays $[11,12]$. We will not discuss this discrepancy of $\left|V_{u s}\right|$ in this work.

${ }^{3}$ The average of the Higgs signal strengths of all production and decay channels from ATLAS and CMS Collaborations are $\mu_{\mathrm{ATLAS}}=1.13_{-0.08}^{+0.09}[15]$, and $\mu_{\mathrm{CMS}}=1.17 \pm 0.10[16]$.
} 
Yukawa coupling at about $6 \%$ from its SM value [17]. Since the $h \rightarrow b \bar{b}$ mode takes up around $58 \%$ of the $125 \mathrm{GeV}$ Higgs total decay width, the above extension can reduce the total Higgs width and universally raise the signal strengths by about $10 \%$ to fit the data.

Finally, the measurement of the forward-backward asymmetry $\mathcal{A}_{F B}^{b}$ of the bottom quark at the $Z^{0}$ pole has exhibited a long-lasting $-2.4 \sigma$ deviation from the SM prediction [9]. Again, this anomaly can be reconciled by introducing an SU(2) doublet VLQs with hypercharge $-5 / 6$. The mixing between the isospin $T_{3}=1 / 2$ component of VLQs and the right-handed SM bottom quark with mixing angle $\sin \theta_{R} \simeq 0.2$ can enhance the right-handed bottom quark coupling with $Z$ boson. Meanwhile, the left-handed bottom quark coupling remains intact [17]. However, the mixing between VLQs and the SM bottom quark is under severe restrictions from other $Z^{0}$-pole observables, for example, the $Z$ hadronic decay width $\Gamma_{\text {had }}$ and the ratio of $Z$ partial width into $b \bar{b}$ relative to the total hadronic width, $R_{b}$, are both consistent with SM predictions. Earlier attempts in this direction can be found in refs. [18, 19].

All the above three discrepancies can be explained with additional heavy quarks, which mix with the SM bottom quark. In order to guarantee the anomaly-free condition, one economical way is to introduce VLQs. The review of various types of VLQs can be found in ref. [20]. In this study, we need to modify both left-handed and right-handed downquark sectors in order to alleviate the above three anomalies. In general, both left-handed and right-handed mixing angles are generated and related to each other for each type of VLQs though one may be suppressed relative to another. It means that we need at least two types of VLQs to simultaneously explain these anomalies. We show that the minimal model requires coexistence of both doublet and singlet VLQs, $\mathcal{B}_{L, R}$ and $b_{L, R}^{\prime \prime}$.

This paper is organized as follows. In section 2, we first write down the general model and study the interactions between VLQs and SM particles, especially the modifications of couplings to $W, Z$, and $h$ bosons. Then we boil down to the requirements of the minimal model. The various constraints from relevant experimental observables are discussed in section 3. In section 4, we perform the chi-square fitting and show numerical results, in particular we discuss the allowed parameter space that can explain all three anomalies. We summarize in section 5 .

\section{Standard model with extra vector-like quarks}

In this work, a doublet and singlet of vector-like quarks (VLQs) are introduced:

$$
\mathcal{B}_{L, R}=\left(\begin{array}{c}
b^{\prime-\frac{1}{3}} \\
p^{\prime-\frac{4}{3}}
\end{array}\right)_{L, R}, \quad b_{L, R}^{\prime \prime-\frac{1}{3}}
$$

with hypercharges $(Y / 2)_{\mathcal{B}_{L, R}}=-5 / 6$ and $(Y / 2)_{b_{L, R}^{\prime \prime}}=-1 / 3$, respectively, under the SM $\mathrm{U}(1)_{Y}$ symmetry. The upper component of the doublet and the singlets have the same quantum numbers as the SM down-type quarks, and thus they are allowed to mix with the SM down-type quarks if nontrivial Yukawa interactions exist among them. It was pointed out that the Yukawa interaction between $\mathcal{B}_{L}$ and $b_{R}$ will induce a mixing between the 
right-handed $b_{R}^{\prime}$ and $b_{R}$, and so reduce the bottom Yukawa coupling. At the same time, it will increase the coupling of the $Z$ boson to the right-handed $b$ quark [17]. The reduction in the bottom Yukawa coupling gives rise to a decrease in the Higgs total decay width, and thus can help alleviate the overall Higgs signal-strength excess, while the increase in the $Z$ coupling to the right-handed $b$ quark can bring the prediction of the forward-backward asymmetry $\mathcal{A}_{F B}^{b}$ down to the experimental value. On the other hand, the mixing between $b_{L}^{\prime}$ and $b_{L}$ is suppressed due to the absence of Yukawa interaction between $\mathcal{B}_{R}$ and $b_{L}$, and so the modification of CKM matrix is negligible. However, the Higgs-induced Yukawa interaction between $b_{L, R}^{\prime \prime}$ and the SM down quarks will give a larger left-handed mixing than the right-handed one. Thus, the non-negligible left-handed mixing can further modify the original $3 \times 3$ CKM matrix and the extra VLQs can extend the CKM matrix to $5 \times 5$ to restore the unitarity.

\subsection{Yukawa couplings and fermion masses}

The generalized interactions between VLQs, SM quarks, and the Higgs doublet are expressed as

$$
\begin{aligned}
-\mathcal{L}_{Y}= & \overline{Q_{L_{i}}^{0}} H \mathbf{y}_{\mathbf{d} i, j} D_{R j}^{0}+\overline{Q_{L_{i}}^{0}} \widetilde{H} \mathbf{y}_{\mathbf{u}_{i, j}} U_{R_{j}}^{0}+\overline{\mathcal{B}_{L}^{0}} \widetilde{H} g_{\mathcal{B} i} D_{R_{i}}^{0}+M_{1} \overline{\mathcal{B}_{L}^{0}} \mathcal{B}_{R}^{0} \\
& +\overline{Q_{L_{i}}^{0}} H g_{b^{\prime \prime} i} b_{R}^{\prime \prime}+M_{2} \overline{b_{L}^{\prime \prime}} b_{R}^{\prime \prime}+m_{5 j} \overline{b_{L}^{\prime \prime}} D_{R j}^{0}+\overline{\mathcal{B}_{L}^{0}} \widetilde{H} g_{\mathcal{B}_{L} b_{R}^{\prime \prime}} b_{R}^{\prime \prime}+\overline{\mathcal{B}_{R}^{0}} \widetilde{H} g_{\mathcal{B}_{R} b_{L}^{\prime \prime}} b_{L}^{\prime \prime}+\text { h.c. }
\end{aligned}
$$

where $U, D$ represent the SM up- and down-quarks with $i, j=1,2,3$ as the flavor indices, and superscript 0 indicates flavor eigenstates, for which the SM Yukawa matrix $\mathbf{y}_{\mathbf{u}, \mathbf{d}}$ have been diagonalized. Note the implicit sum over the repeated indices in the above equation. The dual of Higgs field $\widetilde{H} \equiv i \tau_{2} H^{*}$ carries $Y / 2=-1 / 2$, where $\tau_{2}$ is the Pauli matrix.

After the electroweak symmetry breaking (EWSB), $H=(0, v / \sqrt{2})^{T}$, the mass matrix of the down-type quarks becomes

$$
\left(\begin{array}{lll}
\overline{D^{0}} & \overline{b^{\prime 0}} & \overline{b^{\prime \prime} 0}
\end{array}\right)_{L} \mathcal{M}\left(\begin{array}{c}
D^{0} \\
b^{\prime 0} \\
b^{\prime \prime 0}
\end{array}\right)_{R} \equiv\left(\overline{D^{0}} \overline{b^{\prime 0}} \overline{b^{\prime \prime 0}}\right)_{L}\left(\begin{array}{cccc}
\mathbf{y}_{\mathbf{d}} v / \sqrt{2} & 0 & \xi_{\mathbf{2}} \\
\xi_{1} & M_{1} & \xi_{3} \\
\mathbf{m}_{\mathbf{5}} & \xi_{4} & M_{2}
\end{array}\right)\left(\begin{array}{c}
D^{0} \\
b^{\prime 0} \\
b^{\prime \prime 0}
\end{array}\right)_{R}
$$

where $\xi_{1}=v / \sqrt{2}\left(g_{\mathcal{B} 1}, g_{\mathcal{B} 2}, g_{\mathcal{B} 3}\right)$ is a $1 \times 3$ row vector, $\xi_{\mathbf{2}}=v / \sqrt{2}\left(g_{b^{\prime \prime} 1}, g_{b^{\prime \prime} 2}, g_{b^{\prime \prime} 3}\right)^{T}$ is a $3 \times 1$ column vector, $\xi_{3}=g_{\mathcal{B}_{L} b_{R}^{\prime \prime}} v / \sqrt{2}, \xi_{4}=g_{\mathcal{B}_{R} b_{L}^{\prime \prime}} v / \sqrt{2}$ and $\mathbf{m}_{\mathbf{5}}=\left(m_{51}, m_{52}, m_{53}\right)$ is a $1 \times 3$ row vector.

Since both $\mathcal{M M}^{\dagger}$ and $\mathcal{M}^{\dagger} \mathcal{M}$ are symmetric matrices, they can be diagonalized as

$$
\mathcal{V}_{L} \mathcal{M} \mathcal{M}^{\dagger} \mathcal{V}_{L}^{\dagger}=\mathcal{V}_{R} \mathcal{M}^{\dagger} \mathcal{M} \mathcal{V}_{R}^{\dagger}=\mathcal{M}_{\text {diag }}^{2}=\operatorname{diag}\left(m_{d}^{2}, m_{s}^{2}, m_{b}^{2}, m_{b^{\prime}}^{2}, m_{b^{\prime \prime}}^{2}\right)
$$

and

$$
\left(\begin{array}{c}
D \\
b^{\prime} \\
b^{\prime \prime}
\end{array}\right)_{R, L}=\mathcal{V}_{R, L}\left(\begin{array}{c}
D^{0} \\
b^{\prime 0} \\
b^{\prime \prime 0}
\end{array}\right)_{R, L}
$$


where the mass eigenstates are related to the flavor eigenstates via the unitary matrices $\mathcal{V}_{R, L}$. Similarly, for the up-type quarks the mass eigenstates are related to the flavor eigenstates by

$$
U_{L}=\mathcal{W}_{L} U_{L}^{0}, \quad U_{R}=\mathcal{W}_{R} U_{R}^{0}
$$

Since the VLQs do not mix with up-type quarks, the up-type quark mass matrix remains the same as in SM.

Due to the discrepancies between the mass matrix and Higgs interaction matrix, the Higgs couplings of down-type quarks will be modified from the SM Yukawa couplings,

$$
\begin{aligned}
& -\mathcal{L}_{h} \supset \frac{1}{\sqrt{2}} \overline{D_{L_{i}}^{0}} \mathbf{y}_{\mathbf{d} i, j} D_{R j}^{0} h+\frac{g_{\mathcal{B} i}}{\sqrt{2}} \overline{b_{L}^{0}} D_{R_{i}}^{0} h+\frac{g_{b^{\prime \prime} i}}{\sqrt{2}} \overline{D_{L_{i}}^{0}} b_{R}^{\prime \prime} h+\frac{g_{\mathcal{B}_{L} b_{R}^{\prime \prime}}}{\sqrt{2}} \overline{\mathcal{B}_{L}^{0}} b_{R}^{\prime \prime} h+\frac{g_{\mathcal{B}_{R}} b_{L}^{\prime \prime}}{\sqrt{2}} \overline{\mathcal{B}_{R}^{0}} b_{L}^{\prime \prime} h \\
& + \text { h.c. } \\
& =\left(\overline{D_{L}}, \overline{b_{L}^{\prime}}, \overline{b_{L}^{\prime \prime}}\right) \mathcal{V}_{L}\left(\begin{array}{ccc}
\mathbf{y}_{\mathbf{d}} / \sqrt{2} & 0 & \xi_{\mathbf{2}} / \nu \\
\xi_{\mathbf{1}} / \nu & 0 & \xi_{3} / \nu \\
0 & \xi_{4} / \nu & 0
\end{array}\right) \mathcal{V}_{R}^{\dagger}\left(\begin{array}{c}
D_{L} \\
b_{L}^{\prime} \\
b_{L}^{\prime \prime}
\end{array}\right) h+\text { h.c. } \\
& \equiv\left(\overline{D_{L}}, \overline{b_{L}^{\prime}}, \overline{b_{L}^{\prime \prime}}\right) \mathbf{Y}\left(\begin{array}{c}
D_{L} \\
b_{L}^{\prime} \\
b_{L}^{\prime \prime}
\end{array}\right) h+\text { h.c. }
\end{aligned}
$$

The coupling for $\overline{b_{L}} b_{R} h$ can be extracted out from the matrix element $(\mathbf{Y})_{33}$, for example. Since we only introduce the vector-like quarks that can mix with the bottom quarks, the Higgs couplings to the up-type quarks will stay the same as the SM ones.

\subsection{Modifications to the $W$ couplings with SM quarks}

The charged-current interactions via the $W$ boson with the SM quarks and vector-like quarks are

$$
\begin{aligned}
& -\mathcal{L}_{W} \supset \frac{g_{W}}{\sqrt{2}}\left(\overline{U_{L}}, 0,0\right) \gamma^{\mu} \mathcal{W}_{L} \mathcal{V}_{L}^{\dagger}\left(\begin{array}{c}
D_{L} \\
b_{L}^{\prime} \\
b_{L}^{\prime \prime}
\end{array}\right) W_{\mu}^{+}+\frac{g_{W}}{\sqrt{2}}\left(\overline{D_{L}}, \overline{b_{L}^{\prime}}, \overline{b_{L}^{\prime \prime}}\right) \mathcal{V}_{L} \gamma^{\mu}\left(\begin{array}{c}
\mathbf{0} \\
p_{L}^{\prime} \\
0
\end{array}\right) W_{\mu}^{+} \\
& +\frac{g_{W}}{\sqrt{2}}\left(\overline{D_{R}}, \overline{b_{R}^{\prime}}, \overline{b_{R}^{\prime \prime}}\right) \mathcal{V}_{R} \gamma^{\mu}\left(\begin{array}{c}
\mathbf{0} \\
p_{R}^{\prime} \\
0
\end{array}\right) W_{\mu}^{+}+\text {h.c. } \\
& \equiv \frac{g_{W}}{\sqrt{2}}\left(\bar{U}_{L}, 0,0\right) \gamma^{\mu} \mathbf{V}_{\mathbf{C K M}}^{\mathbf{5} \times \mathbf{5}}\left(\begin{array}{c}
D_{L} \\
b_{L}^{\prime} \\
b_{L}^{\prime \prime}
\end{array}\right) W_{\mu}^{+}+\frac{g_{W}}{\sqrt{2}}\left(\bar{D}, \overline{b^{\prime}}, \overline{b^{\prime \prime}}\right) \gamma^{\mu}\left(\mathcal{V}_{L} P_{L}+\mathcal{V}_{R} P_{R}\right)\left(\begin{array}{c}
\mathbf{0} \\
p^{\prime} \\
0
\end{array}\right) W_{\mu}^{+} \\
& + \text {h.c. }
\end{aligned}
$$

where $P_{L, R}=\frac{1 \mp \gamma_{5}}{2}$. We define the $5 \times 5$ CKM matrix as

$$
\mathbf{V}_{\mathbf{C K M}}^{\mathbf{5} \times \mathbf{5}} \equiv \mathcal{W}_{L} \mathcal{V}_{L}^{\dagger}=\left(\begin{array}{cc}
\left(\mathbf{V}_{\mathbf{C K M}}^{\mathrm{SM}}\right)_{\mathbf{3} \times \mathbf{3}} & 0 \\
0 & \mathbf{1}_{\mathbf{2} \times \mathbf{2}}
\end{array}\right) \mathcal{V}_{L}^{\dagger}
$$


Since the VLQs do not modify the up-quark sector, we simply extend the $3 \times 3$ matrix $\mathcal{W}_{L}$ in eq. (2.9) to a $5 \times 5$ matrix. The exact parameterization of $\mathbf{V}_{\mathbf{C K M}}^{\mathbf{5} \times \mathbf{5}}$ will be shown in appendix A.

We further parameterize the charged current interactions in the following simple form [21],

$$
-\mathcal{L}_{W} \supset \frac{g_{W}}{\sqrt{2}}\left(\overline{q_{L}^{i}} \gamma^{\mu} A_{i j}^{L} q_{L}^{j}+\overline{q_{R}^{i}} \gamma^{\mu} A_{i j}^{R} q_{R}^{j}\right) W_{\mu}^{+}+\text {h.c. }
$$

where $q$ includes all SM quarks and VLQs. $A_{i j}^{L}$ and $A_{i j}^{R}$ are summarized as follows

$$
A_{U_{\alpha} D_{\beta}}^{L}=\left(\mathcal{W}_{L} \mathcal{V}_{L}^{\dagger}\right)_{\alpha \beta}, \quad A_{U_{\alpha} D_{\beta}}^{R}=0, \quad A_{D_{\beta} p^{\prime}}^{L}=\mathcal{V}_{L \beta 4}, \quad A_{D_{\beta} p^{\prime}}^{R}=\mathcal{V}_{R \beta 4}
$$

where $\alpha=1$ to $3, \beta=1$ to 5 , and $\left(U_{1}, U_{2}, U_{3}\right)=(u, c, t),\left(D_{1}, D_{2}, D_{3}, D_{4}, D_{5}\right)=$ $\left(d, s, b, b^{\prime}, b^{\prime \prime}\right)$.

\subsection{Modifications to the $Z$ couplings with the SM quarks}

In the SM, since the couplings between the $Z$ boson and fermions are the same for each generation of up-type and down-type quarks, there are no tree-level flavor-changing neutral currents (FCNC). Conversely, if the new vector-like bottom quarks have different $T_{3 f}-$ $Q_{f} x_{w}$ values from the SM down-type quarks, interesting FCNC couplings can appear at tree level.

According to $T_{3 f}-Q_{f} x_{w}$, the $Z$ boson couplings with the SM down-type quarks and VLQs are

$$
\begin{aligned}
-\mathcal{L} \supset & g_{Z}\left(\bar{D}_{L},{\overline{b^{\prime}}}_{L}, \overline{b^{\prime \prime}} L\right) \gamma^{\mu} \mathcal{V}_{L}\left(\begin{array}{ccc}
-\frac{1}{2}+\frac{1}{3} x_{w} & 0 & 0 \\
0 & \frac{1}{2}+\frac{1}{3} x_{w} & 0 \\
0 & 0 & \frac{1}{3} x_{w}
\end{array}\right) \mathcal{V}_{L}^{\dagger}\left(\begin{array}{c}
D_{L} \\
b_{L}^{\prime} \\
b_{L}^{\prime \prime}
\end{array}\right) Z_{\mu} \\
& +g_{Z}\left(\bar{D}_{R}, \overline{b^{\prime}}{ }_{R}, \overline{b^{\prime \prime}}{ }_{R}\right) \gamma^{\mu} \mathcal{V}_{R}\left(\begin{array}{ccc}
\frac{1}{3} x_{w} & 0 & 0 \\
0 & \frac{1}{2}+\frac{1}{3} x_{w} & 0 \\
0 & 0 & \frac{1}{3} x_{w}
\end{array}\right) \mathcal{V}_{R}^{\dagger}\left(\begin{array}{c}
D_{R} \\
b_{R}^{\prime} \\
b_{R}^{\prime \prime}
\end{array}\right) Z_{\mu} \\
& +g_{Z}{\overline{p^{\prime}}}_{L} \gamma^{\mu}\left(-\frac{1}{2}+\frac{4}{3} x_{w}\right) p_{L}^{\prime} Z_{\mu}+g_{Z}{\overline{p^{\prime}}}_{R} \gamma^{\mu}\left(-\frac{1}{2}+\frac{4}{3} x_{w}\right) p_{R}^{\prime} Z_{\mu},
\end{aligned}
$$

where $Q_{f}\left(T_{3 f}\right)$ is the electric charge (third component of isospin) of quarks, the gauge coupling $g_{Z}=g_{2} / \cos \theta_{w}, x_{w}=\sin ^{2} \theta_{w}$ is the sine-square of the Weinberg angle $\theta_{w}$. Again, the $Z$ boson couplings to the SM up-type quarks are exactly the same as in the SM and are not modified by VLQs.

We further parameterize the $Z$ boson couplings with SM down-type quarks and VLQs in the following simple form [21],

$$
-\mathcal{L}_{Z} \supset \frac{g_{Z}}{2} \bar{q}_{i} \gamma^{\mu}\left[X_{i j}^{L} P_{L}+X_{i j}^{R} P_{R}-2 Q_{i} \delta_{i j} x_{w}\right] q_{j} Z_{\mu},
$$

where $X_{i j}^{L}$ and $X_{i j}^{R}$ are summarized below,

$$
X_{D_{\beta} D_{\beta^{\prime}}}^{L}=-\sum_{i=1}^{3} \mathcal{V}_{L \beta i} \mathcal{V}_{L \beta^{\prime} i}^{*}+\mathcal{V}_{L \beta 4} \mathcal{V}_{L \beta^{\prime} 4}^{*}, \quad X_{D_{\beta} D_{\beta^{\prime}}}^{R}=\mathcal{V}_{R \beta 4} \mathcal{V}_{R \beta^{\prime} 4}^{*}, \quad X_{p^{\prime} p^{\prime}}^{L}=X_{p^{\prime} p^{\prime}}^{R}=1
$$




\subsection{Minimal models}

In this subsection, we would like to narrow down to the most relevant couplings to the experimental anomalies.

First, we consider non-zero couplings $g_{\mathcal{B}_{3}}, g_{b_{1}^{\prime \prime}}$, while $M_{1,2}$ are at TeV scale. According to ref. [17], the tensions of Higgs signal strength and $\mathcal{A}_{\mathrm{FB}}^{b}$ can be alleviated by the $g_{\mathcal{B}_{3}}$ coupling from the doublet VLQ. Then the CKM unitarity violation mainly due to the $\left|V_{u d}\right|$ is relevant to $g_{b_{1}^{\prime \prime}}$ from the singlet VLQ. Other parameters in eq. (2.2) are set to zero. It simplifies the down-type quark mass matrix and $\mathcal{V}_{L, R}$ as

$$
\mathcal{M}=\left(\begin{array}{ccccc}
0 & 0 & 0 & 0 & \bar{\Delta} \\
0 & 0 & 0 & 0 & 0 \\
0 & 0 & m & 0 & 0 \\
0 & 0 & \Delta & M_{1} & 0 \\
0 & 0 & 0 & 0 & M_{2}
\end{array}\right), \quad \mathcal{V}_{L}=\left(\begin{array}{ccccc}
c_{15}^{L} & 0 & 0 & 0 & -s_{15}^{L} \\
0 & 1 & 0 & 0 & 0 \\
0 & 0 & c_{34}^{L} & -s_{34}^{L} & 0 \\
0 & 0 & s_{34}^{L} & c_{34}^{L} & 0 \\
s_{15}^{L} & 0 & 0 & 0 & c_{15}^{L}
\end{array}\right), \quad \mathcal{V}_{R}=\left(\begin{array}{ccccc}
1 & 0 & 0 & 0 & 0 \\
0 & 1 & 0 & 0 & 0 \\
0 & 0 & c_{34}^{R} & -s_{34}^{R} & 0 \\
0 & 0 & s_{34}^{R} & c_{34}^{R} & 0 \\
0 & 0 & 0 & 0 & 1
\end{array}\right),
$$

where $c_{15}^{L, R} \equiv \sqrt{1-\left(s_{15}^{L, R}\right)^{2}}, c_{34}^{L, R} \equiv \sqrt{1-\left(s_{34}^{L, R}\right)^{2}}$, and

$$
s_{34}^{R} \simeq \frac{\Delta}{\sqrt{M_{1}^{2}+\Delta^{2}}}, \quad s_{34}^{L} \simeq \frac{m \Delta}{M_{1}^{2}+\Delta^{2}}, \quad s_{15}^{L}=\frac{\bar{\Delta}}{\sqrt{M_{2}^{2}+\bar{\Delta}^{2}}},
$$

with $\Delta \equiv \frac{g_{\mathcal{B}_{3}} v}{\sqrt{2}}$ and $\bar{\Delta} \equiv \frac{g_{b^{\prime \prime}} v}{\sqrt{2}}$. Here we have taken the liberty that the first two generations of the SM down-type quark masses are set at zero. If the couplings $g_{\mathcal{B}_{3}}, g_{b^{\prime \prime}}{ }_{1}$ are about $\mathcal{O}(1)$, the parameters follow the ordering $M_{1,2}>\Delta, \bar{\Delta} \gg m$. It also implies $s_{34}^{L} \ll s_{34}^{R}$, due to the suppression factor $\mathcal{O}\left(\mathrm{m} / \mathrm{M}_{1}\right)$ on $s_{34}^{L}$. After diagonalizing the mass matrix, the mass of the bottom quark is

$$
m_{b}^{2}=\frac{m^{2}}{1+\left(\Delta^{2} / M_{1}^{2}\right)} .
$$

According to eq. (2.7), the coupling for $(h / v) \bar{b}_{L} b_{R}$ is given by

$$
m c_{34}^{L} c_{34}^{R}-\Delta s_{34}^{L} c_{34}^{R} \simeq m_{b} \frac{c_{34}^{R}}{\sqrt{1+\left(\Delta^{2} / M_{1}^{2}\right)}} .
$$

This gives rise to a reduction factor in the Higgs Yukawa coupling by $C_{h b b} \equiv$ $c_{34}^{R} / \sqrt{1+\left(\Delta^{2} / M_{1}^{2}\right)}$, and thus the enhancement of Higgs signal strengths. The modification of the CKM matrix is indicated by eq. (2.9). The first row of first three elements of $\mathbf{V}_{\mathbf{C K M}}^{\mathbf{5} \times \mathbf{5}}$ violates unitarity as

$$
\left|V_{u d}^{\mathrm{SM}} c_{15}^{L}\right|^{2}+\left|V_{u s}^{\mathrm{SM}}\right|^{2}+\left|V_{u b}^{\mathrm{SM}} c_{34}^{L}\right|^{2}=1-\left|V_{u d}^{\mathrm{SM}}\right|^{2}\left(s_{15}^{L}\right)^{2}-\left|V_{u b}^{\mathrm{SM}}\right|^{2}\left(s_{34}^{L}\right)^{2} .
$$

However, the unitarity for the first row of $\mathbf{V}_{\mathbf{C K M}}^{\mathbf{5} \times \mathbf{5}}$ can be restored with the other two elements

$$
V_{u b^{\prime}}=V_{u b}^{\mathrm{SM}} s_{34}^{L} \quad V_{u b^{\prime \prime}}=V_{u d}^{\mathrm{SM}} s_{15}^{L} .
$$

If $s_{15}^{L} \sim s_{34}^{L}$, we anticipate the contribution from $V_{u b^{\prime \prime}}$ will be dominant. 
Finally, from eq. (2.13) the $Z b b$ couplings are modified as

$$
\left(g^{b}\right)_{L}=g_{Z}\left(-\frac{1}{2}\left(c_{34}^{L}{ }^{2}-s_{34}^{L}{ }^{2}\right)+\frac{1}{3} x_{w}\right), \quad\left(g^{b}\right)_{R}=g_{Z}\left(\frac{1}{2} s_{34}^{R}{ }^{2}+\frac{1}{3} x_{w}\right) .
$$

Since $s_{34}^{R}$ enhances $\left(g^{b}\right)_{R}$, it alleviates the tension between $\mathcal{A}_{F B}^{b}$ observation and SM prediction.

Second, we include one more non-zero coupling $g_{b_{3}^{\prime \prime}}$. Then the mass matrix and unitary transformations matrices are

$$
\begin{aligned}
& \mathcal{M}=\left(\begin{array}{ccccc}
0 & 0 & 0 & 0 & \bar{\Delta} \\
0 & 0 & 0 & 0 & 0 \\
0 & 0 & m & 0 & \Delta^{\prime} \\
0 & 0 & \Delta & M_{1} & 0 \\
0 & 0 & 0 & 0 & M_{2}
\end{array}\right), \\
& \mathcal{V}_{L} \simeq\left(\begin{array}{ccccc}
c_{15}^{L} & 0 & 0 & 0 & -s_{15}^{L} \\
0 & 1 & 0 & 0 & 0 \\
-s_{35}^{L} s_{15}^{L} & 0 & c_{35}^{L} c_{34}^{L} & -c_{35}^{L} s_{34}^{L} & -s_{35}^{L} c_{15}^{L} \\
0 & 0 & s_{34}^{L} & c_{34}^{L} & s_{45}^{L} \\
c_{35}^{L} s_{15}^{L} & 0 & c_{34}^{L} s_{35}^{L} & -s_{45}^{L} & c_{35}^{L} c_{15}^{L}
\end{array}\right), \quad \mathcal{V}_{R} \simeq\left(\begin{array}{ccccc}
1 & 0 & 0 & 0 & 0 \\
0 & 1 & 0 & 0 & 0 \\
0 & 0 & c_{35}^{R} c_{34}^{R} & -c_{35}^{R} s_{34}^{R}-s_{35}^{R} \\
0 & 0 & s_{34}^{R} & c_{34}^{R} & s_{45}^{R} \\
0 & 0 & 0 & -s_{45}^{R} & c_{35}^{R}
\end{array}\right),
\end{aligned}
$$

where $\Delta^{\prime} \equiv \frac{g_{b_{3}^{\prime \prime} v}}{\sqrt{2}}, c_{35}^{L, R} \equiv \sqrt{1-\left(s_{35}^{L, R}\right)^{2}}, c_{45}^{L, R} \equiv \sqrt{1-\left(s_{45}^{L, R}\right)^{2}}$, and

$$
\begin{aligned}
& s_{35}^{R} \simeq \frac{m \Delta^{\prime}}{\sqrt{M_{1}^{2}+\Delta^{\prime 2}}}, \quad s_{35}^{L} \simeq \frac{\Delta^{\prime}}{\sqrt{M_{1}^{2}+\Delta^{\prime 2}}}, \\
& s_{45}^{R} \simeq \frac{m \Delta^{\prime} s_{34}^{R}}{M_{1}^{2}\left(c_{34}^{R}\right)^{2}+2 \Delta M_{1} c_{34}^{R} s_{34}^{R}-\left(\Delta^{\prime 2}+M_{2}^{2}\right)}, \\
& s_{45}^{L} \simeq \frac{\Delta^{\prime} M_{2} s_{34}^{L}}{\left(\Delta^{2}+M_{2}^{1}\right)-M_{2}^{2}\left(c_{35}^{L}\right)^{2}-2 \Delta^{\prime} M_{2} c_{35}^{L} s_{35}^{L}} .
\end{aligned}
$$

Here we diagonalize $\mathcal{M M}^{\dagger}$ via a 4-step block diagonalization procedure. We have used rotation matrices with the order of $R\left(\theta_{15}\right), R\left(\theta_{35}\right), R\left(\theta_{34}\right)$, and $R\left(\theta_{45}\right)$ to block diagonalize $\mathcal{M M}^{\dagger}$ in each step and finally $\mathcal{V}_{L}$ and $\mathcal{V}_{R}$ can be approximated by eq. (2.22). The mass of the bottom quark $m_{b} \simeq m c_{34}^{L}\left(c_{35}^{R} c_{34}^{R} c_{35}^{L}\right)$ and the coupling $(h / v) \bar{b}_{L} b_{R}$ is given by

$$
\simeq\left(m c_{34}^{L}-\Delta s_{34}^{L}\right)\left(c_{35}^{R} c_{34}^{R} c_{35}^{L}\right)=m_{b} \frac{c_{34}^{R}}{\sqrt{1+\left(\Delta^{2} / M_{1}^{2}\right)}}
$$

which is the same as eq. (2.18). The first three elements in the first row of $\mathbf{V}_{\mathbf{C K M}}^{\mathbf{5} \times \mathbf{5}}$ violate unitarity as

$$
1-\left|V_{u b}^{\mathrm{SM}}\right|^{2}\left(s_{34}^{L}\right)^{2}-\left\{\left|V_{u d}^{\mathrm{SM}}\right|^{2}\left(c_{35}^{L}\right)^{2}\left(s_{15}^{L}\right)^{2}+\left|V_{u b}^{\mathrm{SM}}\right|^{2}\left(c_{34}^{L}\right)^{2}\left(s_{35}^{L}\right)^{2}+2 \mathbf{R e}\left[V_{u d}^{\mathrm{SM}} V_{u b}^{\mathrm{SM} *}\right] c_{34}^{L} c_{35}^{L} s_{15}^{L} s_{35}^{L}\right\} .
$$


Similarly, the unitarity in the fist row of $\mathbf{V}_{\mathbf{C K M}}^{\mathbf{5} \times \mathbf{5}}$ can be restore by the other two elements

$$
V_{u b^{\prime}}=V_{u b}^{\mathrm{SM}} s_{34}^{L} \quad V_{u b^{\prime \prime}}=V_{u d}^{\mathrm{SM}} c_{35}^{L} s_{15}^{L}+V_{u b}^{\mathrm{SM}} c_{34}^{L} s_{35}^{L} .
$$

Once again, the contribution from $V_{u b^{\prime \prime}}$ is the dominant one. Then the $Z d d, Z b b, Z d b$ couplings are given by ${ }^{4}$

$$
\begin{array}{rlrl}
\left(g^{d}\right)_{L} & =g_{Z}\left(-\frac{1}{2} c_{15}^{L}{ }^{2}+\frac{1}{3} x_{w}\right), & \left(g^{d}\right)_{R} & =g_{Z}\left(\frac{1}{3} x_{w}\right), \\
\left(g^{b}\right)_{L} & =g_{Z}\left\{-\frac{1}{2}\left[c_{35}^{L}{ }^{2}\left(c_{34}^{L}{ }^{2}-s_{34}^{L}{ }^{2}\right)+s_{35}^{L}{ }^{2} s_{15}^{L}{ }^{2}\right]+\frac{1}{3} x_{w}\right\}, & \left(g^{b}\right)_{R} & =g_{Z}\left(\frac{1}{2} c_{35}^{R^{2}} s_{34}^{R^{2}}+\frac{1}{3} x_{w}\right), \\
\left(g^{d b}\right)_{L} & =g_{Z}\left(\frac{1}{2} s_{35}^{L} s_{15}^{L} c_{15}^{L}\right), & \left(g^{d b}\right)_{R}=0 .
\end{array}
$$

The FCNC is generated from $\left(g^{d b}\right)_{L}$ and shall be constrained by $B_{d^{-}}^{0} \bar{B}_{d}^{0}$ mixing, $B \rightarrow \pi \ell^{+} \ell^{-}$ and $B^{0} \rightarrow \mu^{+} \mu^{-}$. More details are shown in the following sections.

\section{Constraints}

\subsection{CKM measurements}

According to the dispersion relation study with experimental data of neutrino-proton scattering in ref. [5], the inner radiative correction with reduced hadronic uncertainties, $\Delta_{R}^{V}=0.02467(22)$, significantly redetermined the value of $\left|V_{u d}\right|$. The values quoted from PDG 2018 [9] and ref. [4] are

$$
\begin{aligned}
\left|V_{u s}\right| & =0.22333 \pm 0.00060[4] \\
\left|V_{u s} / V_{u d}\right| & =0.23130 \pm 0.00050[4] \\
\left|V_{u d}\right| & =0.97370 \pm 0.00014[4] \\
\left|V_{u b}\right| & =0.00394 \pm 0.00036[9]
\end{aligned}
$$

which we use in our chi-square fitting. As a result, the unitarity condition of the first row of the CKM matrix reads $\left|V_{u d}\right|^{2}+\left|V_{u s}\right|^{2}+\left|V_{u b}\right|^{2}=0.99798 \pm 0.00038$, which deviates from unitarity by more than $4 \sigma[4]$. The respective $b^{\prime}$ and $b^{\prime \prime}$ from the doublet and singlet vector-like bottom quarks can ameliorate the above unitarity problem by extending the CKM to a $5 \times 5$ matrix, then the unitarity requirement becomes $\left|V_{u d}\right|^{2}+\left|V_{u s}\right|^{2}+\left|V_{u b}\right|^{2}+$ $\left|V_{u b^{\prime}}\right|^{2}+\left|V_{u b^{\prime \prime}}\right|^{2}=1 .^{5}$

\section{2 $Z$ boson measurements}

Once the $d, s, b$ couplings to the $Z$ boson are modified, we find that the following observables are modified:

\footnotetext{
${ }^{4}$ The $Z s s$ coupling remains unchanged from the SM value, because we keep the $g_{b_{2}^{\prime \prime}}=0$, and thus no mixing between VLQ and $s$-quark in the following discussion. Non-zero of $g_{b_{2}^{\prime \prime}}$ is strongly constrained by $K-\bar{K}$ mixing observables.

${ }^{5}$ Notice that the contribution from $\left|V_{u b^{\prime}}\right|$ is much more suppressed than $\left|V_{u b^{\prime \prime}}\right|$, so the modification for the CKM unitary mainly comes from $\left|V_{u b^{\prime \prime}}\right|$ in our fitting below.
} 
1. Total hadronic width. At tree level, the change to the decay width into $d \bar{d}, s \bar{s}$, or $b \bar{b}$ is given by

$$
\delta \Gamma_{d, s, b}^{\mathrm{BSM}}=\left[\Gamma_{\text {tree }}^{\mathrm{BSM}, d, s, b}-\Gamma_{\mathrm{tree}}^{\mathrm{SM}, d, s, b}\right]\left(1+\frac{\alpha_{s}\left(M_{Z}\right)}{\pi}\right) .
$$

With this modification, the total hadronic width is changed to

$$
\Gamma_{\mathrm{had}}^{\mathrm{BSM}}=\Gamma_{\mathrm{had}}^{\mathrm{SM}}+\delta \Gamma_{d}^{\mathrm{BSM}}+\delta \Gamma_{s}^{\mathrm{BSM}}+\delta \Gamma_{b}^{\mathrm{BSM}}
$$

2. $\mathbf{R}_{\mathbf{b}}$. The $R_{b}$ is the fraction of hadronic width into $b \bar{b}$, which is given by

$$
R_{b}=\frac{\Gamma_{b}^{\mathrm{SM}}+\delta \Gamma_{b}^{\mathrm{BSM}}}{\Gamma_{\mathrm{had}}^{\mathrm{SM}}+\delta \Gamma_{d}^{\mathrm{BSM}}+\delta \Gamma_{s}^{\mathrm{BSM}}+\delta \Gamma_{b}^{\mathrm{BSM}}} .
$$

3. $\mathcal{A}_{\mathrm{FB}}^{\mathrm{b}}$. There is a large tension in the forward-backward asymmetry of $b$ quark production at the $Z$ resonance between the experimental measurement and the SM prediction,

$$
\mathcal{A}_{\mathrm{FB}}^{b}=\frac{3}{4} \times \frac{\left(g^{e}\right)_{L}^{2}-\left(g^{e}\right)_{R}^{2}}{\left(g^{e}\right)_{L}^{2}+\left(g^{e}\right)_{R}^{2}} \times \frac{\left(g^{b}\right)_{L}^{2}-\left(g^{b}\right)_{R}^{2}}{\left(g^{b}\right)_{L}^{2}+\left(g^{b}\right)_{R}^{2}} .
$$

The couplings of fermions to the $Z$ boson are basically given by $T_{3}-Q x_{w}$ in the SM. For the electron it is simply

$$
\frac{\left(g^{e}\right)_{L}^{2}-\left(g^{e}\right)_{R}^{2}}{\left(g^{e}\right)_{L}^{2}+\left(g^{e}\right)_{R}^{2}}=\frac{\left(-\frac{1}{2}+x_{w}\right)^{2}-x_{w}^{2}}{\left(-\frac{1}{2}+x_{w}\right)^{2}+x_{w}^{2}}
$$

while for the $b$ quark it is

$$
\frac{\left(g^{b}\right)_{L}^{2}-\left(g^{b}\right)_{R}^{2}}{\left(g^{b}\right)_{L}^{2}+\left(g^{b}\right)_{R}^{2}}=\frac{\left(-\frac{1}{2}+\frac{1}{3} x_{w}\right)^{2}-\frac{1}{9} x_{w}^{2}}{\left(-\frac{1}{2}+\frac{1}{3} x_{w}\right)^{2}+\frac{1}{9} x_{w}^{2}}
$$

It was pointed out in ref. [17] that the interaction term $g_{\mathcal{B}_{3}} \overline{\mathcal{B}_{L}^{0}} \widetilde{H} b_{R}^{0}$ from the doublet vector-like quark $\mathcal{B}_{L, R}$ is able to reconcile this tension.

For the second minimal model, where $g_{\mathcal{B}_{3}}, g_{b_{1,2}^{\prime \prime}}$ are non-zero couplings, the modifications of $\left(g^{b}\right)_{L}$ and $\left(g^{b}\right)_{R}$ can be found from eq. (2.27). If we further assume $s_{15}^{L}, s_{34}^{L} \ll 1$, $c_{35}^{R} \simeq 1$ and apply $\left(c_{35}^{L}\right)^{2}=1-\left(s_{35}^{L}\right)^{2},\left(g^{b}\right)_{L}$ and $\left(g^{b}\right)_{R}$ can be simplified as

$$
\begin{aligned}
\left(g^{b}\right)_{L} & =\underbrace{-\frac{g_{Z}}{2}+\frac{g_{Z}}{3} x_{w}}_{g_{L}^{b, S M}}+\underbrace{\frac{g_{Z}}{2}\left(s_{35}^{L}\right)^{2}}_{\delta\left(g^{b}\right)_{L}} . \\
\left(g^{b}\right)_{R} & =\underbrace{\frac{g_{Z}}{3} x_{w}}_{g_{R}^{b, S M}}+\underbrace{\frac{g_{Z}}{2}\left(s_{34}^{R}\right)^{2}}_{\delta\left(g^{b}\right)_{R}} .
\end{aligned}
$$

Both $s_{34}^{R}$ and $s_{35}^{L}$ can reduce the the forward-backward asymmetry $\mathcal{A}_{\mathrm{FB}}^{b}$ of the quark at $Z$-pole. They are good to fit the measured $\mathcal{A}_{\mathrm{FB}}^{b}$ at a lower value from the SM prediction. 
On the other hand, $s_{35}^{L}$ reduces $R_{b}$ but $s_{34}^{R}$ increases $R_{b}$. We can use both to maintain $R_{b}$ at the SM value. This is achieved in the leading order by

$$
2 g_{L}^{b, S M} \delta\left(g^{b}\right)_{L}+2 g_{R}^{b, S M} \delta\left(g^{b}\right)_{R} \approx 0 \Rightarrow\left(-\frac{1}{2}+\frac{1}{3} x_{w}\right)\left(\frac{1}{2}\left(s_{35}^{L}\right)^{2}\right)+\frac{1}{3} x_{w} \frac{1}{2}\left(s_{34}^{R}\right)^{2}=0 .
$$

Therefore, we require $\left(s_{34}^{R}\right)^{2}=\left(\frac{3}{2 x_{w}}-1\right)\left(s_{35}^{L}\right)^{2}$ in order to maintain $R_{b}$ at the SM prediction. A rough estimation is possible by setting $x_{W} \approx \frac{1}{4}$, and so $\left(s_{34}^{R}\right)^{2} \approx 5\left(s_{35}^{L}\right)^{2}$. Unfortunately, we will see from the Fit-2b in section 4 that the B-meson observables are too restrictive to fulfill this relation. Subsequently, mixing angles are chosen to fit the anomaly in $\mathcal{A}_{\mathrm{FB}}^{b}$.

\section{3 $\quad 125 \mathrm{GeV}$ Higgs precision measurements}

The data for the Higgs signal strengths for the combined $7+8 \mathrm{TeV}$ data from ATLAS and CMS [22] and all the most updated $13 \mathrm{TeV}$ data were summarized in ref. [23]. The overall average signal strength is $\mu_{\text {Higgs }}=1.10 \pm 0.05$ [23], which is moderately above the SM prediction. Using a total of 64 data points, the goodness of the SM description for the Higgs data stands at $\chi^{2} /$ d.o.f. $=53.81 / 64$, which gives a goodness of fit 0.814 . A reduction in the total Higgs decay width can provide a better description of the Higgs data with $\chi^{2} /$ d.o.f. $=51.44 / 63$, corresponding to a goodness of fit 0.851 [23]. The $p$-value of the hypothesis of the single-parameter fit $\left(\Delta \Gamma_{\text {tot }}\right)$ equals 0.12 when the SM is the null hypothesis. Although it is not significantly enough to say they are different, it may still give a hint that the single-parameter fit is indeed better than the SM. In this work, the reduction in the Higgs total width is achieved by a slight reduction in the $\mathrm{RH}$ bottom Yukawa coupling which can be found from the matrix element $(\mathbf{Y})_{33}$ in eq. (2.7) and predominately from the doublet vector-like bottom quark interaction term $g_{\mathcal{B}_{3}} \overline{\mathcal{B}_{L}^{0}} \widetilde{H} b_{R}^{0}{ }^{6}$

\subsection{Electro-Weak Precision Observables(EWPOs)}

The Electro-Weak Precision Observables (EWPOs) can be another important indirect constraint for the mixings and masses of the VLQs. The EWPOs can be represented by a set of oblique parameters $S, T$ and $U$. We apply the data from Particles Data Group (PDG) 2018 review [9] with a fixed $U=0$, and the best fits of $S$ and $T$ parameters are

$$
\Delta S=0.02 \pm 0.07, \quad \Delta T=0.06 \pm 0.06
$$

where $\Delta S$ and $\Delta T$ are defined as

$$
\Delta S \equiv S-S_{\mathrm{SM}}, \quad \Delta T \equiv T-T_{\mathrm{SM}}
$$

We consider the $3 \sigma$ allowed regions of $\Delta S$ and $\Delta T$ parameters in our fitting.

\footnotetext{
${ }^{6}$ Once vector-like bottom quarks are heavier than $1 \mathrm{TeV}$, their contributions to $g g \rightarrow h$ and $h \rightarrow \gamma \gamma$ are tiny. We will ignore these effects in our fitting.
} 
The general form of $S$ parameter can be represented as [21, 24, 25]

$$
\begin{aligned}
S= & \frac{N_{c}}{2 \pi} \sum_{i, j}\left\{\left(\left|A_{i j}^{L}\right|^{2}+\left|A_{i j}^{R}\right|^{2}\right) \psi_{+}\left(y_{i}, y_{j}\right)+2 \operatorname{Re}\left(A_{i j}^{L} A_{i j}^{R *}\right) \psi_{-}\left(y_{i}, y_{j}\right)\right. \\
& \left.-\frac{1}{2}\left[\left(\left|X_{i j}^{L}\right|^{2}+\left|X_{i j}^{R}\right|^{2}\right) \chi_{+}\left(y_{i}, y_{j}\right)+2 \operatorname{Re}\left(X_{i j}^{L} X_{i j}^{R *}\right) \chi_{-}\left(y_{i}, y_{j}\right)\right]\right\},
\end{aligned}
$$

where $N_{c}=3, y_{i} \equiv \frac{M_{q_{i}}^{2}}{M_{Z}^{2}}, M_{q_{i}}$ are the quark masses, and $A_{i j}^{L, R}, X_{i j}^{L, R}$ are defined in eqs. (2.11) and (2.14) respectively. On the other hand, the functions inside $S$ are

$$
\begin{aligned}
& \psi_{+}\left(y_{1}, y_{2}\right)=\frac{1}{3}-\frac{1}{9} \log \frac{y_{1}}{y_{2}} \\
& \psi_{-}\left(y_{1}, y_{2}\right)=-\frac{y_{1}+y_{2}}{6 \sqrt{y_{1} y_{2}}} \\
& \chi_{+}\left(y_{1}, y_{2}\right)=\frac{5\left(y_{1}^{2}+y_{2}^{2}\right)-22 y_{1} y_{2}}{9\left(y_{1}-y_{2}\right)^{2}}+\frac{3 y_{1} y_{2}\left(y_{1}+y_{2}\right)-y_{1}^{3}-y_{2}^{3}}{3\left(y_{1}-y_{2}\right)^{3}} \log \frac{y_{1}}{y_{2}} \\
& \chi_{-}\left(y_{1}, y_{2}\right)=-\sqrt{y_{1} y_{2}}\left[\frac{y_{1}+y_{2}}{6 y_{1} y_{2}}-\frac{y_{1}+y_{2}}{\left(y_{1}-y_{2}\right)^{2}}+\frac{2 y_{1} y_{2}}{\left(y_{1}-y_{2}\right)^{3}} \log \frac{y_{1}}{y_{2}}\right] .
\end{aligned}
$$

The contributions from $t$ and $b$ quarks in the SM for the $S$ parameter can be represented as

$$
S_{\mathrm{SM}}=\frac{N_{c}}{6 \pi}\left[1-\frac{1}{3} \log \left(\frac{m_{t}^{2}}{m_{b}^{2}}\right)\right] .
$$

Similarly, the general form of $T$ parameter can be represented as $[21,24,26]$

$$
\begin{aligned}
T= & \frac{N_{c}}{16 \pi s_{W}^{2} c_{W}^{2}} \sum_{i . j}\left\{\left(\left|A_{i j}^{L}\right|^{2}+\left|A_{i j}^{R}\right|^{2}\right) \theta_{+}\left(y_{i}, y_{j}\right)+2 \operatorname{Re}\left(A_{i j}^{L} A_{i j}^{R *}\right) \theta_{-}\left(y_{i}, y_{j}\right)\right. \\
& \left.-\frac{1}{2}\left[\left(\left|X_{i j}^{L}\right|^{2}+\left|X_{i j}^{R}\right|^{2}\right) \theta_{+}\left(y_{i}, y_{j}\right)+2 \operatorname{Re}\left(X_{i j}^{L} X_{i j}^{R *}\right) \theta_{-}\left(y_{i}, y_{j}\right)\right]\right\}
\end{aligned}
$$

where the functions inside $T$ are

$$
\begin{aligned}
& \theta_{+}\left(y_{1}, y_{2}\right)=y_{1}+y_{2}-\frac{2 y_{1} y_{2}}{y_{1}-y_{2}} \log \left(\frac{y_{1}}{y_{2}}\right) \\
& \theta_{-}\left(y_{1}, y_{2}\right)=2 \sqrt{y_{1} y_{2}}\left[\frac{y_{1}+y_{2}}{y_{1}-y_{2}} \ln \left(\frac{y_{1}}{y_{2}}\right)-2\right] .
\end{aligned}
$$

The contributions from $t$ and $b$ quarks in the SM for the $T$ parameter can be represented as

$$
T_{\mathrm{SM}}=\frac{N_{c}}{16 \pi s_{W}^{2} c_{W}^{2}} \theta_{+}\left(y_{t}, y_{b}\right)
$$

\subsection{The mixing of $B_{d}^{0}-\bar{B}_{d}^{0}$}

The non-vanishing Yukawa terms $\overline{Q_{L_{i}}^{0}} H g_{b^{\prime \prime} i} b_{R}^{\prime \prime}$ from the singlet VLQ produce FCNC, predominately among the left-handed down-type quarks with the $Z$ boson. The FCNC coupling $d_{L^{-}} b_{L^{-}} Z$ gives an additional contribution to $B_{d}^{0}-\bar{B}_{d}^{0}$ mixing by exchanging a $Z$ boson 
in $s$-channel. The overall expression including the $\mathrm{SM} t-W$ box diagram and $Z$ boson $\mathrm{FCNC}$ is $[27]$

$$
x_{d}=\frac{2 G_{F}}{3 \sqrt{2}} B_{B} f_{B}^{2} m_{B} \eta_{B} \tau_{B_{d}}\left|U_{s t d-d b}^{2}+U_{d b}^{2}\right| \simeq 1.87 \times 10^{6}\left|U_{s t d-d b}^{2}+U_{d b}^{2}\right|,
$$

where $U_{s t d-d b}^{2}$ is from the SM contribution of top- $W$ box diagram, and $-U_{d b} \equiv \mathcal{V}_{L 35}^{*} \mathcal{V}_{L 15}$ from the $Z$ boson FCNC induced by the singlet VLQ. On the other hand, the FCNC contribution from the doublet VLQ, $\mathcal{V}_{L 34}^{*} \mathcal{V}_{L 14}$, is much smaller than that from the singlet VLQ, because the pattern of the mass matrix which suppresses the left-handed mixing angle for doublet VLQ with down and bottom quarks [17]. The prefactor was obtained by substituting the numerical values: the $\sqrt{B_{B}} f_{B}=225 \pm 9 \mathrm{MeV}$ [9] from lattice calculation; the QCD correction $\eta_{B}=0.55$ [28]; the $B_{d}$ lifetime $\tau_{B_{d}}=1.520(4) \mathrm{ps}=2.31 \times 10^{12} \mathrm{GeV}^{-1}$ and mass $m_{B_{d}}=5.27963(15) \mathrm{GeV}[9]$; and Fermi constant $G_{F}$. The expression for SM contribution is given by [29]

$$
U_{s t d-d b}^{2} \equiv\left(\frac{G_{F} m_{W}^{2}}{2 \sqrt{2} \pi^{2}}\right) y_{t} f_{2}\left(y_{t}\right)\left|V_{t d}^{*} V_{t b}\right|^{2},
$$

where $y_{t} \equiv m_{t}^{2} / m_{W}^{2}$ and the loop function [29]

$$
f_{2}(y) \equiv 1-\frac{3}{4} \frac{y(1+y)}{(1-y)^{2}}\left[1+\frac{2 y}{1-y^{2}} \ln (y)\right]
$$

Taking the most updated experimental values of $\left|V_{t b}\right|=1.019 \pm 0.025$ and $\left|V_{t d}\right|=$ $(8.1 \pm 0.5) \times 10^{-3}[9]$, the SM reproduces the central value of the current experimental measurement $[9]$

$$
\left.x_{d}\right|_{\exp }=0.770 \pm 0.004
$$

However, the theoretical uncertainty is much larger than the experimental one. For conservative limit we require the new physics contribution to be less than the SM contribution, which implies

$$
\left|U_{d b}\right| \leq 6.42 \times 10^{-4}
$$

that is much weaker than the constraints from $B^{+} \rightarrow \pi^{+} \ell^{+} \ell^{-}$and $B^{0} \rightarrow \mu^{+} \mu^{-}$in the next two subsections. In addition, due to large theoretical uncertainties we do not use this data in our global analysis.

On the other hand, the mixings between the second generation quarks and new VLQs are irrelevant in this study. In order to avoid the stringent constraints from the mixing of $D^{0}-\bar{D}^{0}, K^{0}-\bar{K}^{0}$, and $B_{s}^{0}-\bar{B}_{s}^{0}$ mesons, we suppress all the interaction terms between the second generation quarks and new VLQs for simplicity. ${ }^{7}$ The more general study can be found in ref. [32].

\footnotetext{
${ }^{7}$ For this reason we do not attempt to explain the experimental anomalies in $b \rightarrow s l^{+} l^{-}$decays (refs. [33, 34]) in our model.
} 
3.6 The $B^{+} \rightarrow \pi^{+} \ell^{+} \ell^{-}$

The FCNC coupling $\left(g^{d b}\right)_{L}$ generated from eq. (2.27) contributes to the $B^{+} \rightarrow \pi^{+} \ell^{+} \ell^{-}[30]$ through the effective Hamiltonian

$$
\mathcal{H}_{\mathrm{eff}}^{\mathrm{VLQ}}=-\frac{G_{F}}{\sqrt{2}} \frac{\left(g^{d b}\right)_{L}}{g_{z}}\left[\bar{d} \gamma^{\mu}\left(1-\gamma_{5}\right) b\right]\left\{\left(-1+4 x_{w}\right)\left[\bar{\ell} \gamma_{\mu} \ell\right]+\left[\bar{\ell} \gamma_{\mu} \gamma_{5} \ell\right]\right\}
$$

Incorporating with the SM contribution, the differential branching ratio is given by [30]

$$
\begin{aligned}
\frac{d \mathrm{Br}}{d q^{2}}\left(B^{+} \rightarrow \pi^{+} \mu^{+} \mu^{-}\right)= & \frac{G_{F}^{2} M_{B}^{3}}{96 \pi^{3} \Gamma_{B}}\left(\frac{\alpha}{4 \pi}\right)^{2} \lambda\left(q^{2}, m_{\pi}^{2}\right)^{3} \xi_{\pi}^{2}\left(q^{2}\right)\left|\lambda_{t}\right|^{2} \\
& \times\left(\left|\mathcal{C}_{9, P}^{t}+\frac{\lambda_{u}}{\lambda_{t}} \mathcal{C}_{9, P}^{u}+C_{9}^{\mathrm{VLQ}}\right|^{2}+\left|C_{10}+C_{10}^{\mathrm{VLQ}}\right|^{2}\right),
\end{aligned}
$$

with the SM Wilson coefficients $\mathcal{C}_{9, P}^{t} \simeq 3.97+0.03 i, \mathcal{C}_{9, P}^{u} \simeq 0.84-0.88 i$, and $C_{10} \simeq$ -4.25 . Follow the effective operator notations from ref. [30], the VLQs induced Wilson coefficients are

$$
C_{9}^{\mathrm{VLQ}} \equiv \frac{\left(g^{d b}\right)_{L}\left(-1+4 x_{w}\right)}{g_{z}}\left(\frac{2 \pi}{\alpha \lambda_{t}}\right), \quad C_{10}^{\mathrm{VLQ}} \equiv \frac{\left(g^{d b}\right)_{L}}{g_{z}}\left(\frac{2 \pi}{\alpha \lambda_{t}}\right)
$$

here $\lambda_{t} \equiv\left(V_{\mathrm{CKM}}^{\mathrm{SM}}\right)_{t d}\left(V_{\mathrm{CKM}}^{\mathrm{SM}}\right)_{t b}^{*}, \lambda_{u} \equiv\left(V_{\mathrm{CKM}}^{\mathrm{SM}}\right)_{u d}\left(V_{\mathrm{CKM}}^{\mathrm{SM}}\right)_{u b}^{*}, \alpha=1 / 137$, and

$$
\begin{aligned}
\lambda\left(q^{2}, m_{\pi}^{2}\right) & \equiv\left[\left(1-\frac{q^{2}}{M_{B}^{2}}\right)^{2}-\frac{2 m_{\pi}^{2}}{M_{B}^{2}}\left(1+\frac{q^{2}}{M_{B}^{2}}\right)+\frac{m_{\pi}^{4}}{M_{B}^{4}}\right]^{\frac{1}{2}}, \\
\xi\left(q^{2}\right) & \equiv \frac{0.26}{\left(1-q^{2} / M_{B^{*}}^{2}\right)\left(1-0.53 q^{2} / M_{B}^{2}\right)} .
\end{aligned}
$$

The above expression is valid in a conservative range of $1<q^{2}<6 \mathrm{GeV}^{2}$. By performing the integration of the differential branching ratio, we obtain the SM contribution [31]

$$
\operatorname{Br}\left(B^{+} \rightarrow \pi^{+} \mu^{+} \mu^{-}\right)_{\mathrm{SM}}=7.10 \pm 2.13 \times 10^{-9}, \quad q^{2} \subset[1,6] \mathrm{GeV}^{2} .
$$

Within $1 \sigma$ it is consistent with the measurement from $\mathrm{LHCb}$ [35]

$$
\operatorname{Br}\left(B^{+} \rightarrow \pi^{+} \mu^{+} \mu^{-}\right)_{\mathrm{LHCb}}=\left(4.55_{-1.00}^{+1.05} \pm 0.15\right) \times 10^{-9}, \quad q^{2} \subset[1,6] \mathrm{GeV}^{2} .
$$

In the following chi-square fitting, we combine both the experimental error and $30 \%$ theoretical uncertainty from the SM [30] to give conservative constraints.

3.7 The $B^{0} \rightarrow \mu^{+} \mu^{-}$

The $C_{10}^{\mathrm{VLQ}}$ operator also contributes to the $B^{0} \rightarrow \mu^{+} \mu^{-}$through the expression [31]

$$
\operatorname{Br}\left(B^{0} \rightarrow \mu^{+} \mu^{-}\right)=\frac{G_{F}^{2} \alpha^{2}\left|V_{t b}^{*} V_{t d}\right|^{2}}{16 \pi^{3} \Gamma_{B^{0}}} m_{B^{0}} f_{B}^{2} m_{\mu}^{2} \sqrt{1-\frac{4 m_{\mu}^{2}}{m_{B^{0}}^{2}}}\left|C_{10}+C_{10}^{\mathrm{VLQ}}\right|^{2},
$$

where $f_{B}=225 \mathrm{MeV}$. In our framework, the $\left(g^{d b}\right)_{R}=0$ from eq. (2.27) guarantees no mixing among the right-handed $d$ and $b$ quarks and thus $C_{10}^{\prime}$ defined in ref. [31] is zero. 
The updated experimental result from PDG gives [9]

$$
\operatorname{Br}\left(B^{0} \rightarrow \mu^{+} \mu^{-}\right) \operatorname{EXP}=\left(1.4_{-1.4}^{+1.6}\right) \times 10^{-10},
$$

which is consistent with the $\mathrm{SM}$ calculation $\operatorname{Br}\left(B^{0} \rightarrow \mu^{+} \mu^{-}\right)_{\mathrm{SM}}=(1.45 \pm 0.07) \times 10^{-10}$, here we estimated $5 \%$ theoretical uncertainty [31].

\subsection{Direct searches for the vector-like bottom quarks}

The vector-like bottom quarks can be pair produced by QCD processes or singly produced via a $t$-channel $Z$ boson exchange at hadron colliders. Assuming that the new vectorlike bottom quarks can only decay to SM particles, there are three possible decay modes: $b^{\prime}\left(b^{\prime \prime}\right) \rightarrow W^{-} t, b^{\prime}\left(b^{\prime \prime}\right) \rightarrow Z b$, and $b^{\prime}\left(b^{\prime \prime}\right) \rightarrow H b$. The searches for pair production of vectorlike bottom quarks only depend on their masses, decay patterns, and branching ratios. According to ref. [36], the ATLAS Collaboration has published their combined searches for pair production of vector-like bottom quarks with the above three decay modes. The $\mathrm{SU}(2)$ singlet vector-like bottom quark $b^{\prime \prime}$ is excluded for masses below $1.22 \mathrm{TeV}$, and the $\mathrm{SU}(2)$ doublet vector-like bottom quark $\mathcal{B}=\left(b^{-1 / 3}, p^{-4 / 3}\right)^{T}$ is excluded for masses below $1.14 \mathrm{TeV}$. Other recent searches for pair production of vector-like bottom quarks from CMS Collaboration can be found in refs. [37, 38], and those constraints are similar to ref. [36].

On the other hand, the searches for single production of vector-like bottom quarks depend not only on their masses, but also on their mixing with SM down-type quarks. Recently, the ATLAS Collaboration has published their searches for single production of vector-like bottom quark with decays into a Higgs boson and a $b$ quark, followed by $H \rightarrow \gamma \gamma$ in ref. [39]. Again, this constraint is roughly the same as the above ones. Similarly, the searches for pair production and single production of vector-like quark $p^{\prime}$ with electric charge $-4 / 3$ can be found in refs. [40,41]. A lower mass limit about $1.30 \mathrm{TeV}$ at $95 \%$ confidence level is set on the $p^{\prime}$. In order to escape the constraints from these direct searches at the LHC, we can increase $m_{b^{\prime}}, m_{p^{\prime}}$, and $m_{b^{\prime \prime}}$ to be above the lower bounds of the mass constraints. Therefore, we safely set their masses at $1.5 \mathrm{TeV}$ in the analysis.

\section{$4 \quad$ Fitting}

Five data sets are considered in our analysis. Totally, we used 75 data points: 64 from $125 \mathrm{GeV}$ Higgs signal strengths; four from CKM; three from $\mathcal{A}_{\mathrm{FB}}^{b}, R_{b}^{\mathrm{EXP}}, \Gamma_{\text {had }}$ each; two from $\Delta S, \Delta T$; and two from $\operatorname{Br}\left(B^{+} \rightarrow \pi^{+} \ell^{+} \ell^{-}\right)$and $\operatorname{Br}\left(B^{0} \rightarrow \mu^{+} \mu^{-}\right)$. They are summarized in table 1.

The SM CKM matrix is parameterized using the Wolfenstein parameters [9]

$$
V_{\mathrm{CKM}}^{\mathrm{SM}} \equiv\left(\begin{array}{ccc}
1-\lambda^{2} / 2 & \lambda & A \lambda^{3}(\rho-i \eta) \\
-\lambda & 1-\lambda^{2} / 2 & A \lambda^{2} \\
A \lambda^{3}(1-\rho-i \eta) & -A \lambda^{2} & 1
\end{array}\right)
$$

with

$$
\begin{aligned}
\lambda & =0.22453 \pm 0.00044, & A & =0.836 \pm 0.015, \\
\rho & =0.122_{-0.017}^{+0.018}, & \eta & =0.355_{-0.011}^{+0.012},
\end{aligned}
$$




\begin{tabular}{|lll|}
\hline Experimental Data & SM values & $\chi^{2}(\mathrm{SM})$ \\
\hline$\mu_{\mathrm{Higgs}}=1.10 \pm 0.05$ & 1.00 & $53.81[23]$ \\
$\left(\mathcal{A}_{\mathrm{FB}}^{b}\right)$ & $0.1030 \pm 0.0002$ & $5.29[9]$ \\
$R_{b}^{\mathrm{EXP}}=0.0992 \pm 0.0016$ & $0.21582 \pm 0.00002$ & $0.49[9]$ \\
$\Gamma_{\text {had }}=1.7444 \pm 0.0020 \mathrm{GeV}$ & $1.7411 \pm 0.0008$ & $2.35[9]$ \\
$\mathrm{CKM}:\left|V_{u s}\right|=0.22333 \pm 0.00060$ & $0.22453 \pm 0.00044$ & $24.50[4,9]$ \\
$\left|V_{u s} / V_{u d}\right|=0.23130 \pm 0.00050$ & $0.23041 \pm 0.00045$ & \\
$\left|V_{u d}\right|=0.97370 \pm 0.00014$ & $0.97446 \pm 0.00010$ & \\
$\left|V_{u b}\right|=0.00394 \pm 0.00036$ & $0.00365 \pm 0.00012$ & \\
$\operatorname{EWPOs}: \Delta S=0.02 \pm 0.07$ & 0 & $1.08[9]$ \\
$\Delta T=0.06 \pm 0.06$ & 0 & \\
$\left.\operatorname{Br}\left(B^{+} \rightarrow \pi^{+} \ell^{+} \ell^{-}\right)\right|_{q^{2} \subset[1,6] \mathrm{GeV}^{2}=\left(4.55_{-1.00}^{+1.05} \pm 0.15\right) \times 10^{-9}}$ & $(7.10 \pm 2.13) \times 10^{-9}$ & $1.15[35]$ \\
$\operatorname{Br}\left(B^{0} \rightarrow \mu^{+} \mu^{-}\right)=\left(1.4_{-1.4}^{+1.6}\right) \times 10^{-10}$ & $(1.45 \pm 0.07) \times 10^{-10}$ & $0.00[31]$ \\
\hline
\end{tabular}

Table 1. Experimental data used in the current analysis: (i) the overall Higgs-signal strength representing 64 individual channels of signal strengths, (ii) $3 Z$-pole observables $\mathcal{A}_{\mathrm{FB}}^{b}, R_{b}$ and $\Gamma_{\text {had }}$, (iii) four data from the CKM matrix, (iv) $\Delta S$ and $\Delta T$ from EWPOs, and (v) branching ratios of $B^{+} \rightarrow \pi^{+} \ell^{+} \ell^{-}$and $B^{0} \rightarrow \mu^{+} \mu^{-}$. Note that the $B_{d}^{0}-\bar{B}_{d}^{0}$ mixing data is not used in this analysis.

quoted from the global fit [9]. The SM values of $\left|V_{u s}^{\mathrm{SM}}\right|,\left|V_{u s}^{\mathrm{SM}} / V_{u d}^{\mathrm{SM}}\right|,\left|V_{u d}^{\mathrm{SM}}\right|$, and $\left|V_{u b}\right|$ are listed in table 1, and the uncertainties from global fit in SM are included in our chisquare analysis. In fact, the SM does not fit well to the above datasets, as it gives a total $\chi^{2}(\mathrm{SM}) /$ d.o.f. $=88.946 / 75$, which is translated into a goodness of fit only 0.130 . Note that during the parameter scan, the unitarity condition of $\sum_{i=d, s, b, b^{\prime}, b^{\prime \prime}}\left|V_{u i}\right|^{2}=1$ is always held from our analytical parameterization. The unitary violation only happens on $\sum_{i=d, s, b}\left|V_{u i}\right|^{2}$.

According to the minimal model of additional VLQs with various options on the parameters in subsection 2.4, we perform several fittings to investigate if these models can provide better explanations for the data. Without loss of generality we fix the VLQs mass at $1.5 \mathrm{TeV}$, which is above the current VLQs mass lower bounds from ATLAS and CMS searches [36, 39, 41-44].

- Fit-1: varying $g_{\mathcal{B}_{3}}$ and $g_{b_{1}^{\prime \prime}}$ while keeping $g_{b_{3}^{\prime \prime}}=0, M_{1}=M_{2}=1.5 \mathrm{TeV}$.

- Fit-2a: varying $g_{\mathcal{B}_{3}}, g_{b_{1}^{\prime \prime}}$ and $g_{b_{3}^{\prime \prime}}$ while keeping $M_{1}=M_{2}=1.5 \mathrm{TeV}$. But NOT including the constraints $B^{+} \rightarrow \pi^{+} \ell^{+} \ell^{-}$and $B^{0} \rightarrow \mu^{+} \mu^{-}$in the $\chi^{2}$ fitting.

- Fit-2b: same as Fit-2a, but including the constraint $B^{+} \rightarrow \pi^{+} \ell^{+} \ell^{-}$and $B^{0} \rightarrow \mu^{+} \mu^{-}$ in the $\chi^{2}$ fitting.

For Fit-1, keeping $g_{b_{3}^{\prime \prime}}=0$ can guarantees the flavor-changing coupling $\left(g^{d b}\right)_{L}$ from eq. (2.27) to be zero. Therefore the constraints from $B_{d}^{0} \bar{B}_{d}^{0}$ mixing, $B^{+} \rightarrow \pi^{+} \ell^{+} \ell^{-}$, and $B^{0} \rightarrow \mu^{+} \mu^{-}$are irrelevant. Both the values of $\operatorname{Br}\left(B^{+} \rightarrow \pi^{+} \ell^{+} \ell^{-}\right)$and $\operatorname{Br}\left(B^{0} \rightarrow \mu^{+} \mu^{-}\right)$ are exactly the same as the SM predictions. After performing the fit to the data, Fit-1 gives a minimal chi-square value of $\chi_{\min }^{2} /$ d.o.f. $=63.124 / 73$ and thus a goodness of fit 

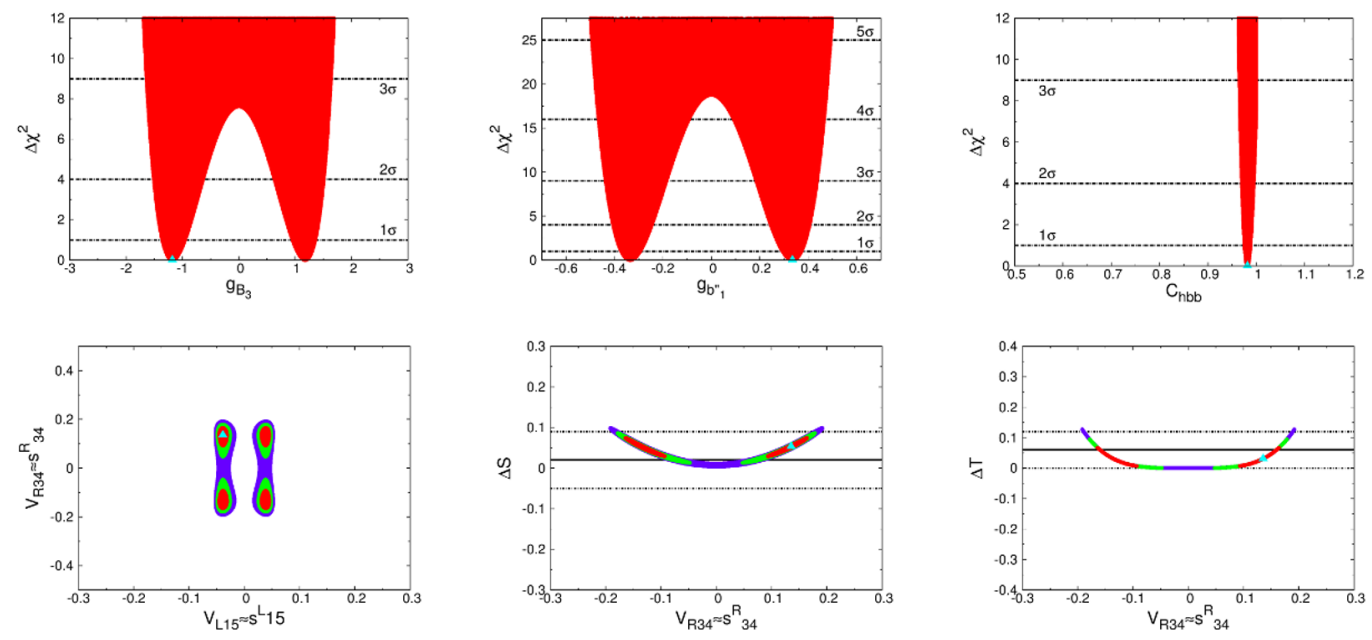

Figure 1. Fit-1: the best fit (cyan triangle) gives $\chi_{\min }^{2}=63.124$. The contour panels show regions for $\Delta \chi^{2} \leq 2.3$ (red), 5.99 (green), and 11.83 (blue) above the minimum.

$=0.789$. Comparing with the SM fit Fit-1 has a $p$-value of $2.5 \times 10^{-6}$ against the SM null hypothesis. It is shown in both table 2 and figure 1 that the best-fit points prefer a non-zero value of $g_{\mathcal{B}_{3}}= \pm 1.177$ and $g_{b_{1}^{\prime \prime}}= \pm 0.335$ at a level more than $2.5 \sigma$ and $4 \sigma$ from zero, respectively. Furthermore, the bottom-quark Yukawa coupling deviates from the SM prediction by more than $2 \sigma$, and the best-fit points give $C_{h b b}=0.98$, which is about $2 \%$ smaller than the SM value. It helps to enhance the overall Higgs signal strengths. In fact, the Higgs signal-strength dataset prefers bottom Yukawa coupling 6\% smaller than the SM value [17]. Since the $R_{b}^{\mathrm{EXP}}$ was quite precisely measured and consistent with the SM prediction, the deviation of the bottom-Yukawa coupling cannot exceed more than a couple of percent. From the $\left(\mathcal{V}_{\mathrm{L} 15}, \mathcal{V}_{\mathrm{R} 34}\right)$ panel of figure 1 , since $\mathcal{V}_{\mathrm{L} 15} \simeq s_{15}^{L} \propto g_{b_{1}^{\prime \prime}}$ and $\mathcal{V}_{\mathrm{R} 34} \simeq s_{34}^{R} \propto g_{\mathcal{B}_{3}}$, it does not show correlation between $g_{\mathcal{B}_{3}}$ and $g_{b_{1}^{\prime \prime}}$. In the $\left(\mathcal{V}_{R 34}, \Delta S\right)$ and $\left(\mathcal{V}_{R 34}, \Delta T\right)$ panels, they show that the best-fit regions are consistent with the oblique parameters from electroweak precision measurements.

In Fit-2, both couplings $g_{b_{1}^{\prime \prime}}$ and $g_{b_{3}^{\prime \prime}}$ can vary from zero. In this case, according to eq. (2.27), flavor-changing coupling $\left(g^{d b}\right)_{L}$ is induced and therefore is constrained $B^{+} \rightarrow$ $\pi^{+} \ell^{+} \ell^{-}$and $B^{0} \rightarrow \mu^{+} \mu^{-}\left(B_{d}^{0} \bar{B}_{d}^{0}\right.$ mixing is not included in any of the fits.) In figure 2 for Fit-2a, which has not included these flavor-changing constraints in the global fit, it allows both couplings $g_{b_{1}^{\prime \prime}}$ and $g_{b_{3}^{\prime \prime}}$ to significantly deviate from zero. Indeed, we see that the best-fit points prefer $g_{\mathcal{B}_{3}}= \pm 1.651$ and $g_{b_{3}^{\prime \prime}}= \pm 0.614$, and $\left(s_{34}^{R}\right)^{2} \simeq 5\left(s_{35}^{L}\right)^{2}$ are correlated in $\left(\mathcal{V}_{L 35}, \mathcal{V}_{R 34}\right)$ panel. This is in accordance with our discussion at end of subsection 3.2, where the VLQs contributions to $R_{b}$ cancel among themselves, meanwhile $\mathcal{A}_{F B}^{b}$ anomaly is explained by $\left(g^{b}\right)_{L}$. Since the VLQs contributions to $R_{b}$ are canceled, the bottom-Yukawa coupling now is allowed to deviate from the SM by more than $6 \%$, and the best-fit points give $C_{h b b}=0.96$, which deviates form the SM prediction by more than $3 \sigma$. Hence, Fit-2a can further lower the minimal chi-square than Fit-1, and gives $\chi_{\min }^{2} /$ d.o.f. $=59.185 / 70$ and thus a goodness of fit equals to 0.818. Unfortunately, there exist constraints from $B_{d}^{0}-\bar{B}_{d}^{0}$ 

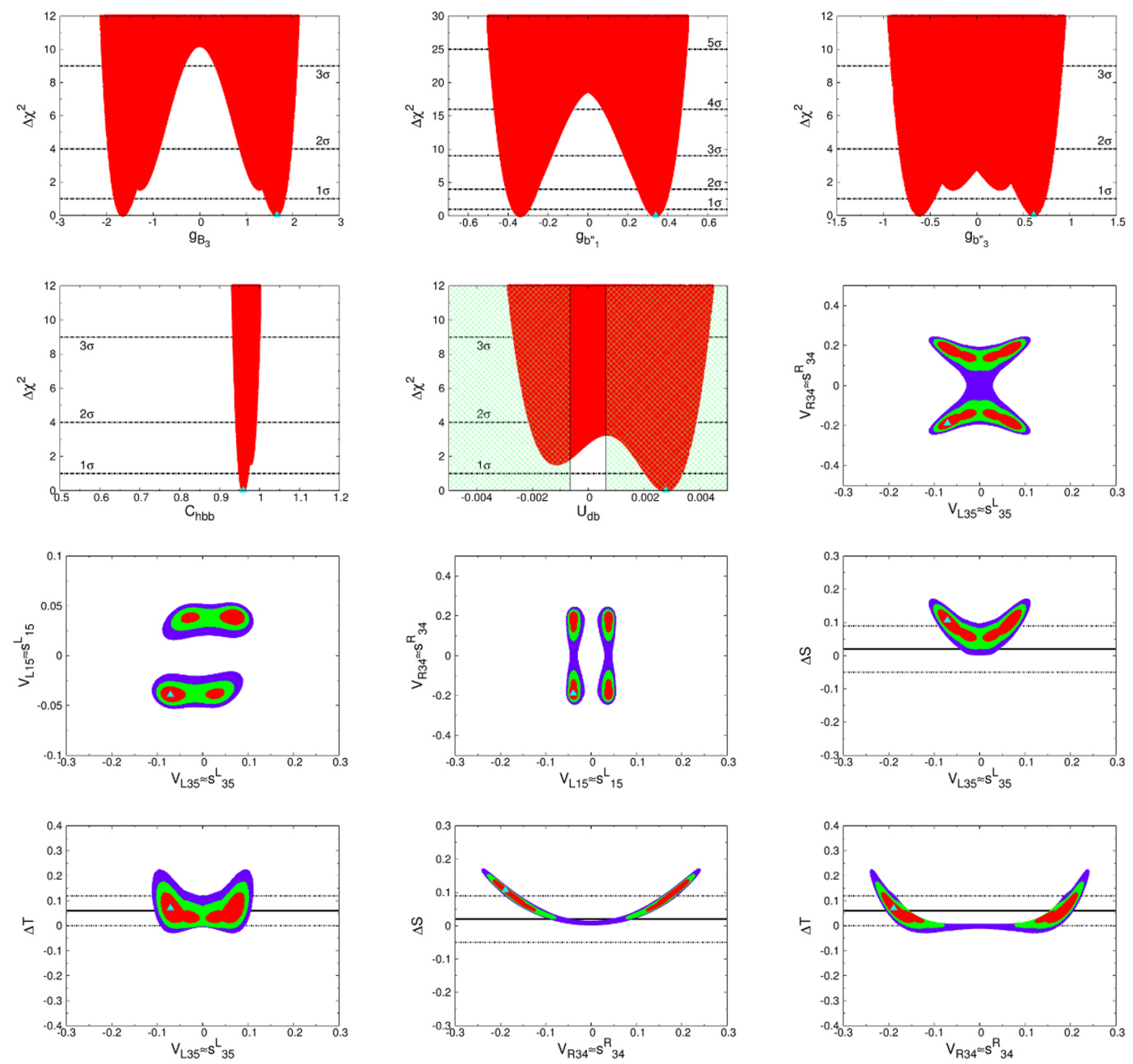

Figure 2. Fit-2a: the best fit (cyan triangle) gives $\chi_{\min }^{2}=59.185$. In $U_{d b}-\Delta \chi^{2}$ panel, the hatched region is excluded by $B_{d}^{0}-\bar{B}_{d}^{0}$ mixing. The contour panels show regions for $\Delta \chi^{2} \leq 2.3$ (red), 5.99 (green), and 11.83 (blue) above the minimum.

mixing, $B^{+} \rightarrow \pi^{+} \ell^{+} \ell^{-}$and $B^{0} \rightarrow \mu^{+} \mu^{-}$, which will restrict simultaneously large non-zero values of $g_{b_{1}^{\prime \prime}}$ and $g_{b_{3}^{\prime \prime}}$. In order to study the effects from those B physics constraints, we further include both $B^{+} \rightarrow \pi^{+} \ell^{+} \ell^{-}$and $B^{0} \rightarrow \mu^{+} \mu^{-}$in the Fit-2b.

In figure 3 for $\mathbf{F i t - 2} \mathbf{b}$, we can understand how the constraints from $B^{+} \rightarrow \pi^{+} \ell^{+} \ell^{-}$ and $B^{0} \rightarrow \mu^{+} \mu^{-}$affect the allowed parameter region. In the $\left(g_{b_{3}^{\prime \prime}}, \Delta \chi^{2}\right)$ panel, the coupling $g_{b_{3}^{\prime \prime}}$ is restricted to be small within $3 \sigma$, more precisely, it requires $\left|g_{b_{3}^{\prime \prime}}\right| \leq 0.076$. Since $g_{b_{3}^{\prime \prime}}$ is restricted close to zero, the best-fit points and the corresponding $C_{h b b}$ of $\mathbf{F i t - 2 \mathbf { b }}$ overlap with Fit-1. In the same panel, we can observe there are two local minima at $g_{b_{3}^{\prime \prime}} \simeq \pm 0.6$ at $4 \sigma$, which is correlated to $g_{b_{1}^{\prime \prime}} \simeq 0$ in $\left(g_{b_{1}^{\prime \prime}}, \Delta \chi^{2}\right)$ panel. From the $\left(U_{d b}, \Delta \chi^{2}\right)$ panel, we know that the flavor constraints from $B^{+} \rightarrow \pi^{+} \ell^{+} \ell^{-}$is more stringent than $B_{d}^{0} \bar{B}_{d}^{0}$ mixing due to more precise theoretical uncertainty in the former. Around the minimum, we can identify the two-tine fork shape structure, and it is due to the interference between 

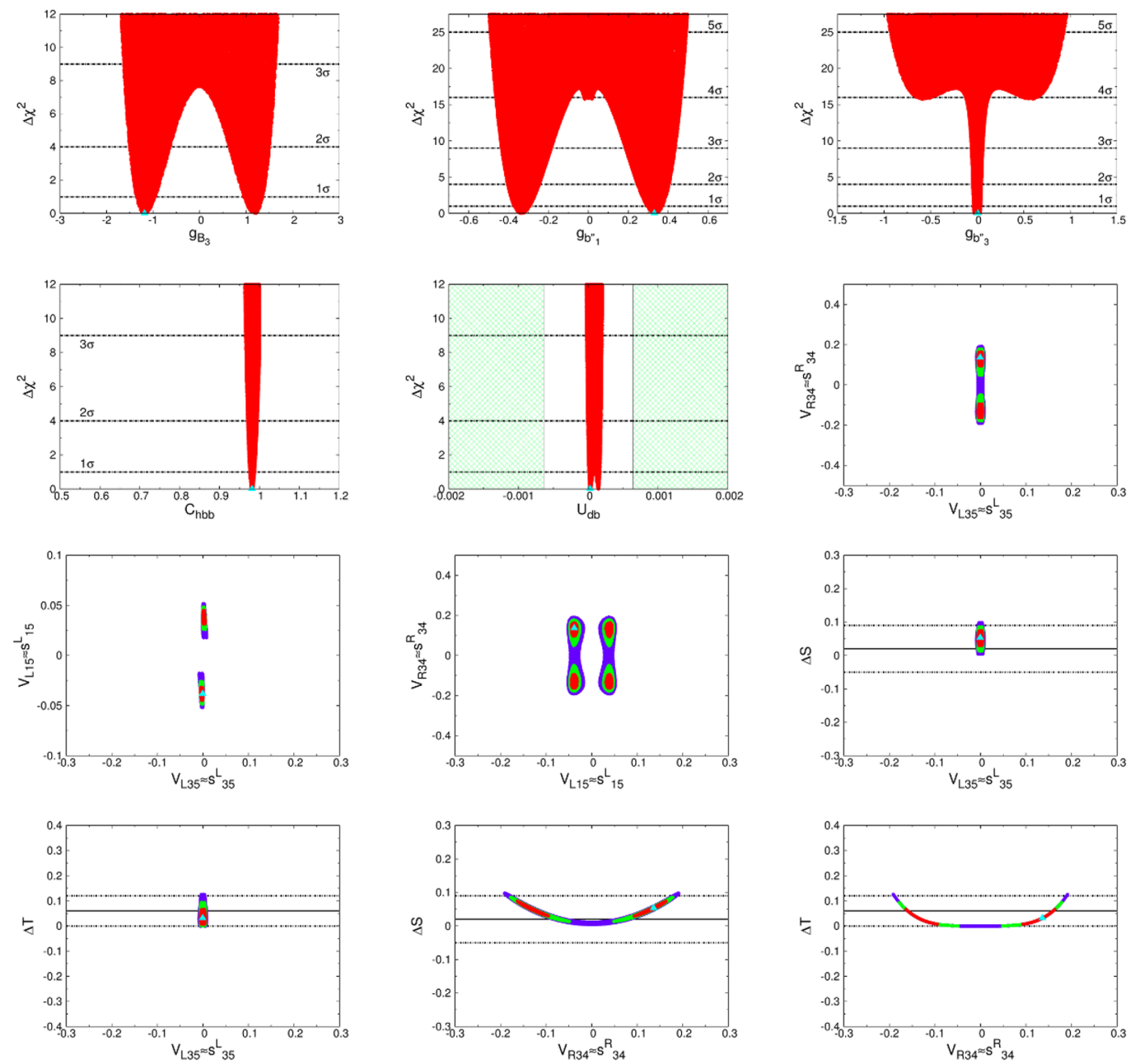

Figure 3. Fit-2b: the best fit (cyan triangle) gives $\chi_{\min }^{2}=62.275$. In $U_{d b}-\Delta \chi^{2}$ panel, the hatched region is excluded by $B_{d}^{0}-\bar{B}_{d}^{0}$ mixing. The contour panels show regions for $\Delta \chi^{2} \leq 2.3$ (red), 5.99 (green), and 11.83 (blue) above the minimum.

VLQs and SM contributions for $B^{+} \rightarrow \pi^{+} \ell^{+} \ell^{-}$from eq. (3.20). Finally, comparing with $B^{+} \rightarrow \pi^{+} \ell^{+} \ell^{-}$, the $B^{0} \rightarrow \mu^{+} \mu^{-}$gives similar but weaker constraint on $\left(g^{d b}\right)_{L}$. We can also find in table 2 that both the values of $\operatorname{Br}\left(B^{+} \rightarrow \pi^{+} \ell^{+} \ell^{-}\right)$and $\operatorname{Br}\left(B^{0} \rightarrow \mu^{+} \mu^{-}\right)$in Fit-2b are largely reduced by three orders of magnitude compared with Fit-2a. On the other hand, we observe that the value of $\operatorname{Br}\left(B^{+} \rightarrow \pi^{+} \ell^{+} \ell^{-}\right)$in Fit-2 $\mathbf{b}$ is indeed closer to the measurement from $\mathrm{LHCb}$ in eq. (3.24) than the SM prediction in eq. (3.23), because the central value in eq. (3.23) is more than $1 \sigma$ larger than the central value in eq. (3.24). Once both theoretical and experimental uncertainties are reduced in the future with almost the same central value in $\operatorname{Br}\left(B^{+} \rightarrow \pi^{+} \ell^{+} \ell^{-}\right)$, it will be a smoking-gun signature for adding VLQs to the SM.

For discovery prospects of the doublet+singlet VLQs, there are some signatures which can be searched for at the LHC. The VLQs can be pair produced via QCD processes, such 


\begin{tabular}{|c|ccc|}
\hline Cases & Fit-1 & Fit-2a & Fit-2b \\
\hline & Vary $g_{\mathcal{B}_{3}}, g_{b_{1}^{\prime \prime}}$ & Vary $g_{\mathcal{B}_{3}}, g_{b_{1}^{\prime \prime}}$ & Vary $g_{\mathcal{B}_{3}}, g_{b_{1}^{\prime \prime}}$ \\
Parameters & & $g_{b_{3}^{\prime \prime}}$ & $g_{b_{3}^{\prime \prime}}$ \\
\hline$g_{\mathcal{B}_{3}}$ & $1.177_{-0.225}^{+0.179}$ & $1.651_{-0.213}^{+0.166}$ & $1.176_{-0.225}^{+0.179}$ \\
$g_{b_{1}^{\prime \prime}}$ & $0.335_{-0.041}^{+0.037}$ & $0.339_{-0.039}^{+0.035}$ & $0.335_{-0.041}^{+0.037}$ \\
$g_{b_{3}^{\prime \prime}}$ & 0 & $0.614_{-0.149}^{+0.113}$ & $0.0063_{-0.0092}^{+0.0049}$ \\
$M_{1}[\mathrm{TeV}]$ & 1.5 & 1.5 & 1.5 \\
$M_{2}[\mathrm{TeV}]$ & 1.5 & 1.5 & 1.5 \\
\hline$C_{h b b}$ & $0.982_{-0.007}^{+0.006}$ & $0.960_{-0.009}^{+0.010}$ & $0.982_{-0.007}^{+0.006}$ \\
$\chi_{\mathrm{Higgs}}^{2}$ & 52.46 & 51.38 & 52.46 \\
$\mathcal{A}_{\mathrm{FB}}^{b}$ & 0.10129 & 0.09943 & 0.10129 \\
$R_{b}$ & 0.21732 & 0.21676 & 0.21732 \\
$\Gamma_{\text {tot }}$ & 1.7428 & 1.7415 & 1.7428 \\
$\Delta S$ & 0.05 & 0.11 & 0.05 \\
$\Delta T$ & 0.03 & 0.07 & 0.03 \\
$\operatorname{Br}\left(B^{+} \rightarrow \pi^{+} \ell^{+} \ell^{-}\right)$ & $7.10 \times 10^{-9}$ & $3.87 \times 10^{-6}$ & $4.92 \times 10^{-9}$ \\
$\operatorname{Br}\left(B^{0} \rightarrow \mu^{+} \mu^{+}\right)$ & $1.45 \times 10^{-10}$ & $1.36 \times 10^{-7}$ & $0.74 \times 10^{-10}$ \\
\hline \hline$\chi^{2} /$ dof & $63.124 / 73$ & $59.185 / 70$ & $62.275 / 72$ \\
$\operatorname{goodness~of~fit~}$ & 0.789 & 0.818 & 0.786 \\
$p$-value & $2.5 \times 10^{-6}$ & $2.7 \times 10^{-6}$ & $1.2 \times 10^{-5}$ \\
\hline
\end{tabular}

Table 2. The best-fitted values in various fits and the corresponding chi-square per degree of freedom and goodness of fit. The $p$-value for each fit hypothesis against the SM null hypothesis is also shown. For the SM, we obtain $\chi^{2}(\mathrm{SM})=88.946, \chi^{2} /$ dof $=88.946 / 75$, and corresponding goodness of fit $=0.130$. Notice the condition $\sum_{i=d, s, b, b^{\prime}, b^{\prime \prime}}\left|V_{u i}\right|^{2}=1$ is held during the fitting.

as $g g, q \bar{q} \rightarrow b^{\prime} \overline{b^{\prime}}, b^{\prime \prime} \bar{b}^{\prime \prime}, p^{\prime} \bar{p}^{\prime}$. Due to the off-diagonal Yukawa interactions and mixing between VLQs and SM quarks, the VLQs can decay via

$$
\begin{aligned}
b^{\prime} & \rightarrow Z b, Z s, Z d, h b, h s, h d \\
b^{\prime \prime} & \rightarrow W t, W c, W u \\
p^{\prime} & \rightarrow W b, W s, W d .
\end{aligned}
$$

Here, we assume the mass degeneracy of $b^{\prime}$ and $p^{\prime}$ from the doublet VLQ to avoid the decay mode $b^{\prime} \rightarrow p^{\prime} W$ or $p^{\prime} \rightarrow b^{\prime} W$. Even though there is slight mass splitting between $b^{\prime}$ and $p^{\prime}$ of order $\mathcal{O}(10) \mathrm{GeV}$ due to the mixing effect, the decay $p^{\prime} \rightarrow b^{\prime} W$ or $b^{\prime} \rightarrow p^{\prime} W$ can only give very soft leptons or jets, which are very difficult for detection at the LHC.

The decay branching ratios of VLQs, for example, from the best-fit points for Fit-1 and Fit-2b from table 2 are

$$
\begin{aligned}
\mathrm{BR}\left(b^{\prime} \rightarrow Z b\right) & \simeq \mathrm{BR}\left(b^{\prime} \rightarrow h b\right) \simeq 0.5, & \mathrm{BR}\left(p^{\prime} \rightarrow W b\right) & \simeq 1.00 \\
\mathrm{BR}\left(b^{\prime \prime} \rightarrow W c\right) & =0.05, & \operatorname{BR}\left(b^{\prime \prime} \rightarrow W u\right) & =0.95
\end{aligned}
$$


and for Fit-2a,

$$
\begin{array}{rlrl}
\mathrm{BR}\left(b^{\prime} \rightarrow Z b\right) & \simeq \mathrm{BR}\left(b^{\prime} \rightarrow h b\right) \simeq 0.5, & & \mathrm{BR}\left(p^{\prime} \rightarrow W b\right) \simeq 1.00 \\
\mathrm{BR}\left(b^{\prime \prime} \rightarrow W t\right) & =0.765, & & \\
\mathrm{BR}\left(b^{\prime \prime} \rightarrow W c\right) & =0.005, & \mathrm{BR}\left(b^{\prime \prime} \rightarrow W u\right)=0.230,
\end{array}
$$

The above relation $\mathrm{BR}\left(b^{\prime} \rightarrow Z b\right) \simeq \mathrm{BR}\left(b^{\prime} \rightarrow h b\right)$ comes from the equivalence theorem, in which the longitudinal mode of gauge bosons behaves like the Goldstone boson in the limit $M_{b^{\prime}, b^{\prime \prime}, p^{\prime}} \gg m_{Z, h}$. Therefore, one clear signature at the LHC from pair produced $b^{\prime} b^{\prime}$ is

$$
b^{\prime} \bar{b}^{\prime} \rightarrow(b X)(\bar{b} Z) \rightarrow(b X)\left(\bar{b} \ell^{+} \ell^{-}\right),
$$

where $X$ could be either $h$ or $Z$. Such charged lepton pair(s) plus jets searches have been performed at the $13 \mathrm{TeV}$ LHC [37, 44]. Here we roughly estimate the current sensitivity on the lower mass limit of $b^{\prime}$. The event rate with at least one charged lepton pair is

$$
N=\sigma\left(p p \rightarrow b^{\prime} \bar{b}^{\prime}\right) \times \mathcal{L} \times \epsilon
$$

where $\epsilon=0.0028$ taking into account the branching ratios of $b^{\prime}$ and $Z \rightarrow \ell^{+} \ell^{-}$. Then using $\mathcal{L}=36.1 \mathrm{fb}^{-1}$ and requiring $N<2$, we obtain

$$
\sigma\left(p p \rightarrow b^{\prime} \bar{b}^{\prime}\right) \lesssim 20 \mathrm{fb}
$$

By adopting the VLQ pair production cross section, the above upper limit translates into the lower mass limit of $M_{b^{\prime}} \gtrsim 1.1 \mathrm{TeV}$.

Other decay modes of VLQs from pair production have been searched for by ATLAS and CMS Collaborations in refs. [36, 38, 42, 43]. The lower mass limits of VLQs are around $1 \mathrm{TeV}$ from these searches. Single VLQ production via the electroweak interaction, which depends on the size of mixing between VLQ and SM quark, was investigated in refs. [41, 45]. We emphasize the predicted $g_{\mathcal{B}_{3}}$ and $g_{b_{1}^{\prime \prime}}$ values in table II all give $s_{34}^{R} \simeq$ $g_{\mathcal{B}_{3}} v /\left(\sqrt{2} M_{1}\right) \simeq 0.14$ and $s_{15}^{L} \simeq g_{b_{1}^{\prime \prime}} v /\left(\sqrt{2} M_{2}\right) \simeq 0.04$, that can be measured from the single VLQ productions via $Z b(W u)$ fusion and ready to be tested in the near future. For example, the single $p^{\prime}$ produced from the $W b$ fusion has been studied by the ATLAS [41]. Assuming $\operatorname{BR}\left(p^{\prime} \rightarrow W b\right)=100 \%$ and varying $\left|s_{34}^{R}\right|$ between 0.17 and 0.55 , the lower mass limit of $p^{\prime}$ can be set from 800 to $1800 \mathrm{GeV}$.

A distinctive signature of our proposed model from other phenomenological models is the singlet VLQ decay mode $b^{\prime \prime} \rightarrow W^{-} u$. On the other hand, most of experimental searches at the LHC were focused on the mixing between VLQs and the third generation quarks. Hence, we stress the searches for the mixing between VLQs and the first generation quarks are also well-motivated in this work. The sizeable or dominant $\mathrm{BR}\left(b^{\prime \prime} \rightarrow W^{-} u\right)$ can be a distinguishable feature of our scenario.

\section{Discussion}

We have advocated an extension of the SM with vector-like quarks, including a doublet and a singlet, in aim of alleviating a few experimental anomalies. An urgent one is a severe 
unitarity violation in the first row of the CKM matrix standing at a level more than $4 \sigma$ due to a recent more precise evaluation of $V_{u d}$ and $V_{u s}$. Another one is the long-lasting discrepancy in the forward-backward asymmetry $\mathcal{A}_{\mathrm{FB}}^{b}$ in $Z \rightarrow b \bar{b}$ at LEP. Furthermore, a mild excess in the overall Higgs signal strength appears at about $2 \sigma$ above the standard model (SM) prediction,

In this work, we have performed global fits of the model under the constraints coming from the unitarity condition of the first row of the CKM matrix, the $Z$-pole observables $\mathcal{A}_{\mathrm{FB}}^{b}, R_{b}$ and $\Gamma_{\text {had }}$, Electro-Weak precision observables $\Delta S$ and $\Delta T, B$-meson observables $B_{d}^{0}-\bar{B}_{d}^{0}$ mixing, $B^{+} \rightarrow \pi^{+} \ell^{+} \ell^{-}$and $B^{0} \rightarrow \mu^{+} \mu^{-}$, and direct searches for VLQs at the LHC. We found that the extension with a VLQ doublet and a singlet can improve the fitting to the datasets, especially the improvement to the unitarity condition of the first row of the CKM matrix with two additional entries in the first row.

We offer the following comments before closing.

1. By extending the CKM matrix to $5 \times 5$ with the extra VLQs, the unitarity condition in the first row is fully restored.

2. Without taking into account the B-meson constraints the best-fit (see Fit-2a) can allow the bottom-Yukawa coupling to decrease by about $6 \%$, which can then adequately explain the $2 \sigma$ excess in the Higgs signal strength. At the same time, it can also account for the $\mathcal{A}_{\mathrm{FB}}^{b}$ without upsetting $R_{b}$ due to a nontrivial cancellation between two contributions. However, the resulting branching ratios for $B^{+} \rightarrow \pi^{+} \ell^{+} \ell^{-}$ and $B^{0} \rightarrow \mu^{+} \mu^{-}$become exceedingly large above the experimental values.

3. However, including the B-meson constraints the allowed parameter space in $g_{b_{3}^{\prime \prime}}$ is restricted to be very small due to the presence of the FCNC in $Z-b-d$.

4. Last but not least, the extra 5 physical CP phases in $\mathbf{V}_{\mathbf{C K M}}^{\mathbf{5} \times \mathbf{5}}$ matrix can be a trigger for electroweak baryogenesis. In order to generate the strong first-order electroweak phase transition, one needs to add an extra singlet complex scalar [47, 48]. On the other hand, adding extra $Z^{\prime}$ boson as in the ref. [31] would be possible to cancel the FCNC contributions from VLQs. Therefore, a gauge U(1) extension of our minimal model with a singlet complex scalar may simultaneously alleviate the constraints from B meson observables and explain the matter-antimatter asymmetry of the Universe. However, this extension is beyond the scope of this work and we would like to study this possibility in the future.

\section{Acknowledgments}

We thank Jae Sik Lee for initial participation and Chien-Yeah Seng, Michael J. RamseyMusolf for the information about the recent determination of $V_{u d}$. W.-Y. K. and P.-Y. T. thank the National Center of Theoretical Sciences, Taiwan, R.O.C. for hospitality. The work of K.C. was supported by the National Science Council of Taiwan under Grants No. MOST-107-2112-M-007-029-MY3. 


\section{A Parameterization of the full $V_{\mathrm{CKM}}^{5 \times 5}$ matrix}

In this appendix, we display parameterization of the full $\mathbf{V}_{\mathbf{C K M}}^{\mathbf{5} \times \mathbf{5}}$ matrix in the main text. For the general $n \times n$ CKM matrix, there are $n^{2}-(2 n-1)=(n-1)^{2}$ physical parameters in the corresponding matrix. For example, there are 3 rotation angles $\theta_{12}, \theta_{13}, \theta_{23}$ and 1 $\mathrm{CP}$ phase $\delta$ in the $3 \times 3 \mathrm{CKM}$ matrix of SM. For the $5 \times 5 \mathrm{CKM}$ matrix, there are 16 physical parameters. Except for the previous 4 parameters in the $3 \times 3 \mathrm{CKM}$ matrix, we assign the extra 12 parameters as 7 rotation angles $\theta_{14}, \theta_{15}, \theta_{24}, \theta_{25}, \theta_{34}, \theta_{35}, \theta_{45}$ and $5 \mathrm{CP}$ phases $\phi_{1}, \phi_{2}, \phi_{3}, \phi_{4}, \phi_{5}$ in the $5 \times 5$ CKM matrix.

We first parameterize the original $3 \times 3$ CKM matrix in the usual form

$$
\begin{aligned}
\mathbf{V}_{\mathbf{C K M}}^{\mathbf{3} \times \mathbf{3}} & =\left(\begin{array}{ccc}
V_{u d} & V_{u s} & V_{u b} \\
V_{c d} & V_{c s} & V_{c b} \\
V_{t d} & V_{t s} & V_{t b}
\end{array}\right) \\
& =\left(\begin{array}{ccc}
1 & 0 & 0 \\
0 & c_{23} & s_{23} \\
0 & -s_{23} & c_{23}
\end{array}\right)\left(\begin{array}{ccc}
c_{13} & 0 & s_{13} e^{-i \delta} \\
0 & 1 & 0 \\
-s_{13} e^{i \delta} & 0 & c_{13}
\end{array}\right)\left(\begin{array}{ccc}
c_{12} & s_{12} & 0 \\
-s_{12} & c_{12} & 0 \\
0 & 0 & 1
\end{array}\right) \\
& =\left(\begin{array}{cccc}
-s_{12} c_{23}-c_{12} s_{13} s_{23} e^{i \delta} & c_{12} c_{23}-s_{12} s_{13} s_{23} e^{i \delta} & s_{13} e^{-i \delta} \\
s_{12} s_{23}-c_{12} s_{13} c_{23} e^{i \delta} & -c_{12} s_{23}-s_{12} s_{13} c_{23} e^{i \delta} & c_{23} c_{13}
\end{array}\right),
\end{aligned}
$$

with $s_{i j}=\sin \theta_{i j}$ and $c_{i j}=\cos \theta_{i j}$ [46]. Then we can further parameterize the full $5 \times 5$ CKM matrix based on $\mathbf{V}_{\mathbf{C K M}}^{\mathbf{3} \times \mathbf{3}}$ as

$$
\begin{aligned}
& \mathbf{V}_{\mathbf{C K M}}^{\mathbf{5} \times \mathbf{5}}=\left(\begin{array}{ccccc}
V_{u d} & V_{u s} & V_{u b} & V_{u b^{\prime}} & V_{u b^{\prime \prime}} \\
V_{c d} & V_{c s} & V_{c b} & V_{c b^{\prime}} & V_{c b^{\prime \prime}} \\
V_{t d} & V_{t s} & V_{t b} & V_{t b^{\prime}} & V_{t b^{\prime \prime}} \\
V_{t^{\prime} d} & V_{t^{\prime} s} & V_{t^{\prime} b} & V_{t^{\prime} b^{\prime}} & V_{t^{\prime} b^{\prime \prime}} \\
V_{t^{\prime \prime} d} & V_{t^{\prime \prime} s} & V_{t^{\prime \prime} b} & V_{t^{\prime \prime} b^{\prime}} & V_{t^{\prime \prime} b^{\prime \prime}}
\end{array}\right) \\
& =\left(\begin{array}{ccccc}
\mathbf{V}_{\mathbf{C K M}}^{\mathbf{3} \times \mathbf{3}} & & 0 & 0 \\
& & & 0 & 0 \\
0 & 0 & 0 & 1 & 0 \\
0 & 0 & 0 & 0 & 1
\end{array}\right)\left(\begin{array}{ccccc}
1 & 0 & 0 & 0 & 0 \\
0 & 1 & 0 & 0 & 0 \\
0 & 0 & 1 & 0 & 0 \\
0 & 0 & 0 & c_{45} & s_{45} e^{-i \phi_{5}} \\
0 & 0 & 0 & -s_{45} e^{i \phi_{5}} & c_{45}
\end{array}\right)\left(\begin{array}{ccccc}
1 & 0 & 0 & 0 & 0 \\
0 & 1 & 0 & 0 & 0 \\
0 & 0 & 0 & 1 & 0 \\
0 & 0 & c_{35} & 0 & s_{35} \\
0 & 0 & -s_{35} & 0 & c_{35}
\end{array}\right) \\
& \cdot\left(\begin{array}{ccccc}
1 & 0 & 0 & 0 & 0 \\
0 & 1 & 0 & 0 & 0 \\
0 & 0 & c_{34} & s_{34} & 0 \\
0 & 0 & -s_{34} & c_{34} & 0 \\
0 & 0 & 0 & 0 & 1
\end{array}\right)\left(\begin{array}{ccccc}
1 & 0 & 0 & 0 & 0 \\
0 & c_{25} & 0 & 0 & s_{25} e^{-i \phi_{4}} \\
0 & 0 & 1 & 0 & 0 \\
0 & 0 & 0 & 1 & 0 \\
0 & -s_{25} e^{i \phi_{4}} & 0 & 0 & c_{25}
\end{array}\right)\left(\begin{array}{ccccc}
1 & 0 & 0 & 0 & 0 \\
0 & c_{24} & 0 & s_{24} e^{-i \phi_{3}} & 0 \\
0 & 0 & 1 & 0 & 0 \\
0 & -s_{24} e^{i \phi_{3}} & 0 & c_{24} & 0 \\
0 & 0 & 0 & 0 & 1
\end{array}\right) \\
& \cdot\left(\begin{array}{ccccc}
c_{15} & 0 & 0 & 0 & s_{15} e^{-i \phi_{2}} \\
0 & 1 & 0 & 0 & 0 \\
0 & 0 & 1 & 0 & 0 \\
0 & 0 & 0 & 1 & 0 \\
-s_{15} e^{-i \phi_{2}} & 0 & 0 & 0 & c_{15}
\end{array}\right)\left(\begin{array}{cccccc}
c_{14} & 0 & 0 & s_{14} e^{-i \phi_{1}} & 0 \\
0 & 1 & 0 & 0 & 0 \\
0 & 0 & 1 & 0 & 0 \\
-s_{14} e^{i \phi_{1}} & 0 & 0 & c_{14} & 0 \\
0 & 0 & 0 & 0 & 1
\end{array}\right)
\end{aligned}
$$


where

$$
\begin{aligned}
& V_{u d}=c_{14}\left(c_{12} c_{13} c_{15}-e^{i \phi_{2}} s_{15}\left(e^{-i \delta} c_{25} s_{13} s_{35}+e^{-i \phi_{4}} c_{13} s_{12} s_{25}\right)\right) \\
& -e^{i \phi_{1}} s_{14}\left(e^{-i \phi_{3}} s_{24}\left(c_{13} c_{25} s_{12}-e^{i\left(\phi_{4}-\delta\right)} s_{13} s_{25} s_{35}\right)+e^{-i \delta} c_{24} c_{35} s_{13} s_{34}\right) \\
& V_{u s}=c_{24}\left(c_{13} c_{25} s_{12}-e^{i\left(\phi_{4}-\delta\right)} s_{13} s_{25} s_{35}\right)-e^{i\left(\phi_{3}-\delta\right)} c_{35} s_{13} s_{24} s_{34} \\
& V_{u b}=e^{-i \delta} c_{34} c_{35} s_{13} \\
& V_{u b^{\prime}}=e^{-i \phi_{1}} s_{14}\left(c_{12} c_{13} c_{15}-e^{i \phi_{2}} s_{15}\left(e^{-i \delta} c_{25} s_{13} s_{35}+e^{-i \phi_{4}} c_{13} s_{12} s_{25}\right)\right) \\
& +c_{14}\left(e^{-i \phi_{3}} s_{24}\left(c_{13} c_{25} s_{12}-e^{i\left(\phi_{4}-\delta\right)} s_{13} s_{25} s_{35}\right)+e^{-i \delta} c_{24} c_{35} s_{13} s_{34}\right) \\
& V_{u b^{\prime \prime}}=c_{15}\left(e^{-i \delta} c_{25} s_{13} s_{35}+e^{-i \phi_{4}} c_{13} s_{12} s_{25}\right)+e^{-i \phi_{2}} c_{12} c_{13} s_{15} \\
& V_{c d}=-c_{14}\left(c_{15}\left(c_{23} s_{12}+e^{i \delta} c_{12} s_{13} s_{23}\right)+e^{i \phi_{2}} s_{15}\left(c_{13} c_{25} s_{23} s_{35}+e^{-i \phi_{4}} s_{25}\left(c_{12} c_{23}-e^{i \delta} s_{12} s_{13} s_{23}\right)\right)\right) \\
& -e^{i \phi_{1}} s_{14}\left(c_{13} c_{24} c_{35} s_{23} s_{34}+e^{-i \phi_{3}} s_{24}\left(c_{25}\left(c_{12} c_{23}-e^{i \delta} s_{12} s_{13} s_{23}\right)-e^{i \phi_{4}} c_{13} s_{23} s_{25} s_{35}\right)\right) \\
& V_{c s}=c_{24}\left(c_{25}\left(c_{12} c_{23}-e^{i \delta} s_{12} s_{13} s_{23}\right)-e^{i \phi_{4}} c_{13} s_{23} s_{25} s_{35}\right)-e^{i \phi_{3}} c_{13} c_{35} s_{23} s_{24} s_{34} \\
& V_{c b}=c_{13} c_{34} c_{35} s_{23} \\
& V_{c b^{\prime}}=-e^{-i \phi_{1}} s_{14}\left(c_{15}\left(c_{23} s_{12}+e^{i \delta} c_{12} s_{13} s_{23}\right)\right. \\
& \left.+e^{i \phi_{2}} s_{15}\left(c_{13} c_{25} s_{23} s_{35}+e^{-i \phi_{4}} s_{25}\left(c_{12} c_{23}-e^{i \delta} s_{12} s_{13} s_{23}\right)\right)\right) \\
& +c_{14}\left(c_{13} c_{24} c_{35} s_{23} s_{34}+e^{-i \phi_{3}} s_{24}\left(c_{25}\left(c_{12} c_{23}-e^{i \delta} s_{12} s_{13} s_{23}\right)-e^{i \phi_{4}} c_{13} s_{23} s_{25} s_{35}\right)\right) \\
& V_{c b^{\prime \prime}}=-e^{-i \phi_{2}} s_{15}\left(c_{23} s_{12}+e^{i \delta} c_{12} s_{13} s_{23}\right)+c_{15}\left(c_{13} c_{25} s_{23} s_{35}+e^{-i \phi_{4}} s_{25}\left(c_{12} c_{23}-e^{i \delta} s_{12} s_{13} s_{23}\right)\right) \\
& V_{t d}=c_{14}\left(c_{15}\left(s_{12} s_{23}-e^{i \delta} c_{12} c_{23} s_{13}\right)-e^{i \phi_{2}} s_{15}\left(c_{13} c_{23} c_{25} s_{35}-e^{-i \phi_{4}} s_{25}\left(c_{12} s_{23}+e^{i \delta} c_{23} s_{12} s_{13}\right)\right)\right) \\
& -e^{i \phi_{1}} s_{14}\left(c_{13} c_{23} c_{24} c_{35} s_{34}-e^{-i \phi_{3}} s_{24}\left(c_{25}\left(c_{12} s_{23}+e^{i \delta} c_{23} s_{12} s_{13}\right)+e^{i \phi_{4}} c_{13} c_{23} s_{25} s_{35}\right)\right) \\
& V_{t s}=-c_{24}\left(c_{25}\left(c_{12} s_{23}+e^{i \delta} c_{23} s_{12} s_{13}\right)+e^{i \phi_{4}} c_{13} c_{23} s_{25} s_{35}\right)-e^{i \phi_{3}} c_{13} c_{23} c_{35} s_{24} s_{34} \\
& V_{t b}=c_{13} c_{23} c_{34} c_{35} \\
& V_{t b^{\prime}}=e^{-i \phi_{1}} s_{14}\left(c_{15}\left(s_{12} s_{23}-e^{i \delta} c_{12} c_{23} s_{13}\right)\right. \\
& \left.-e^{i \phi_{2}} s_{15}\left(c_{13} c_{23} c_{25} s_{35}-e^{-i \phi_{4}} s_{25}\left(c_{12} s_{23}+e^{i \delta} c_{23} s_{12} s_{13}\right)\right)\right) \\
& +c_{14}\left(c_{13} c_{23} c_{24} c_{35} s_{34}-e^{-i \phi_{3}} s_{24}\left(c_{25}\left(c_{12} s_{23}+e^{i \delta} c_{23} s_{12} s_{13}\right)-e^{i \phi_{4}} c_{13} c_{23} s_{25} s_{35}\right)\right) \\
& V_{t b^{\prime \prime}}=e^{-i \phi_{2}} s_{15}\left(s_{12} s_{23}-e^{i \delta} c_{12} c_{23} s_{13}\right)+c_{15}\left(c_{13} c_{23} c_{25} s_{35}-e^{-i \phi_{4}} s_{25}\left(c_{12} s_{23}+e^{i \delta} c_{23} s_{12} s_{13}\right)\right) \\
& V_{t^{\prime} d}=-e^{i\left(\phi_{2}-\phi_{5}\right)} c_{14} c_{25} c_{35} s_{15} s_{45}-e^{i \phi_{1}} s_{14}\left(c_{24}\left(c_{34} c_{45}-e^{-i \phi_{5}} s_{34} s_{35} s_{45}\right)\right. \\
& \left.-e^{i\left(\phi_{4}-\phi_{3}-\phi_{5}\right)} c_{35} s_{24} s_{25} s_{45}\right) \\
& V_{t^{\prime} s}=-e^{i\left(\phi_{4}-\phi_{5}\right)} c_{24} c_{35} s_{25} s_{45}-e^{i \phi_{3}} s_{24}\left(c_{34} c_{45}-e^{-i \phi_{5}} s_{34} s_{35} s_{45}\right) \\
& V_{t^{\prime} b}=-c_{45} s_{34}-e^{-i \phi_{5}} c_{34} s_{35} s_{45} \\
& V_{t^{\prime} b^{\prime}}=c_{14}\left(c_{24}\left(c_{34} c_{45}-e^{-i \phi_{5}} s_{34} s_{35} s_{45}\right)-e^{i\left(\phi_{4}-\phi_{3}-\phi_{5}\right)} c_{35} s_{24} s_{25} s_{45}\right)-e^{i\left(\phi_{2}-\phi_{1}-\phi_{5}\right)} c_{25} c_{35} s_{14} s_{15} s_{45} \\
& V_{t^{\prime} b^{\prime \prime}}=e^{-i \phi_{5}} c_{15} c_{25} c_{35} s_{45} \\
& V_{t^{\prime \prime} d}=-e^{i \phi_{2}} c_{14} c_{25} c_{35} c_{45} s_{15}+e^{i \phi_{1}} s_{14}\left(c_{24}\left(c_{45} s_{34} s_{35}+e^{i \phi_{5}} c_{34} s_{45}\right)+e^{i\left(\phi_{4}-\phi_{3}\right)} c_{35} c_{45} s_{24} s_{25}\right) \\
& V_{t^{\prime \prime} s}=-e^{i \phi_{4}} c_{24} c_{35} c_{45} s_{25}+e^{i \phi_{3}} s_{24}\left(c_{45} s_{34} s_{35}+e^{i \phi_{5}} c_{34} s_{45}\right) \\
& V_{t^{\prime \prime} b}=-c_{34} c_{45} s_{35}+e^{i \phi_{5}} s_{34} s_{45} \\
& V_{t^{\prime \prime} b^{\prime}}=-c_{14}\left(c_{24}\left(c_{45} s_{34} s_{35}+e^{i \phi_{5}} c_{34} s_{45}\right)+e^{i\left(\phi_{4}-\phi_{3}\right)} c_{35} c_{45} s_{24} s_{25}\right)-e^{i\left(\phi_{2}-\phi_{1}\right)} c_{25} c_{35} c_{45} s_{14} s_{15} \\
& V_{t^{\prime \prime} b^{\prime \prime}}=c_{15} c_{25} c_{35} c_{45}
\end{aligned}
$$


Notice that there is some freedom to arrange the positions of extra $5 \mathrm{CP}$ phases in those matrices. We assign there is no $\mathrm{CP}$ phase in the rotation matrices of $\theta_{34}$ and $\theta_{35}$ in this study. On the other hand, since we don't involve the vector-like up-type quarks $t^{\prime}, t^{\prime \prime}$ inside the model, only the measurable $3 \times 5$ sub-matrix of $\mathbf{V}_{\mathbf{C K M}}^{\mathbf{5} \times \mathbf{5}}$ is corresponding for our study here.

Open Access. This article is distributed under the terms of the Creative Commons Attribution License (CC-BY 4.0), which permits any use, distribution and reproduction in any medium, provided the original author(s) and source are credited.

\section{References}

[1] N. Cabibbo, Unitary Symmetry and Leptonic Decays, Phys. Rev. Lett. 10 (1963) 531 [INSPIRE].

[2] M. Kobayashi and T. Maskawa, CP Violation in the Renormalizable Theory of Weak Interaction, Prog. Theor. Phys. 49 (1973) 652 [InSPIRE].

[3] J.C. Hardy and I.S. Towner, Superallowed $0^{+} \rightarrow 0^{+}$nuclear $\beta$ decays: 2014 critical survey, with precise results for $V_{u d}$ and CKM unitarity, Phys. Rev. C 91 (2015) 025501 [arXiv:1411.5987] [INSPIRE].

[4] B. Belfatto, R. Beradze and Z. Berezhiani, The CKM unitarity problem: A trace of new physics at the TeV scale?, Eur. Phys. J. C 80 (2020) 149 [arXiv:1906.02714] [INSPIRE].

[5] C.-Y. Seng, M. Gorchtein, H.H. Patel and M.J. Ramsey-Musolf, Reduced Hadronic Uncertainty in the Determination of $V_{u d}$, Phys. Rev. Lett. 121 (2018) 241804 [arXiv: 1807.10197] [INSPIRE].

[6] C.Y. Seng, M. Gorchtein and M.J. Ramsey-Musolf, Dispersive evaluation of the inner radiative correction in neutron and nuclear $\beta$ decay, Phys. Rev. D 100 (2019) 013001 [arXiv: 1812.03352] [INSPIRE].

[7] C.-Y. Seng and U.-G. Meissner, Toward a First-Principles Calculation of Electroweak Box Diagrams, Phys. Rev. Lett. 122 (2019) 211802 [arXiv: 1903.07969] [INSPIRE].

[8] M. Moulson, Experimental determination of $V_{u s}$ from kaon decays, PoS (CKM2016) 033 (2017) [arXiv: 1704.04104] [INSPIRE].

[9] Particle Data Group collaboration, Review of Particle Physics, Phys. Rev. D 98 (2018) 030001.

[10] A.M. Coutinho, A. Crivellin and C.A. Manzari, Global Fit to Modified Neutrino Couplings and the Cabibbo-Angle Anomaly, arXiv:1912.08823 [INSPIRE].

[11] HFLAV collaboration, Averages of b-hadron, c-hadron and $\tau$-lepton properties as of 2018, arXiv:1909.12524 [INSPIRE].

[12] A. Lusiani, HFLAV $\tau$ branching fractions fit and measurements of $\left|V_{u s}\right|$ with $\tau$ lepton data, SciPost Phys. Proc. 1 (2019) 001 [arXiv:1811.06470] [INSPIRE].

[13] ATLAS collaboration, Observation of a new particle in the search for the Standard Model Higgs boson with the ATLAS detector at the LHC, Phys. Lett. B 716 (2012) 1 [arXiv: 1207.7214] [INSPIRE]. 
[14] CMS collaboration, Observation of a New Boson at a Mass of $125 \mathrm{GeV}$ with the CMS Experiment at the LHC, Phys. Lett. B 716 (2012) 30 [arXiv:1207.7235] [INSPIRE].

[15] ATLAS collaboration, Combined measurements of Higgs boson production and decay using up to $80 \mathrm{fb}^{-1}$ of proton-proton collision data at $\sqrt{s}=13 \mathrm{TeV}$ collected with the ATLAS experiment, ATLAS-CONF-2018-031 (2018).

[16] CMS collaboration, Combined measurements of Higgs boson couplings in proton-proton collisions at $\sqrt{s}=13 \mathrm{TeV}$, Eur. Phys. J. C 79 (2019) 421 [arXiv:1809.10733] [InSPIRE].

[17] K. Cheung, W.-Y. Keung, J.S. Lee and P.-Y. Tseng, Vector-like Quark Interpretation of Excess in Higgs Signal Strength, Phys. Lett. B 798 (2019) 134983 [arXiv:1901.05626] [INSPIRE].

[18] D. Choudhury, T.M.P. Tait and C.E.M. Wagner, Beautiful mirrors and precision electroweak data, Phys. Rev. D 65 (2002) 053002 [hep-ph/0109097] [InSPIRE].

[19] B. Batell, S. Gori and L.-T. Wang, Higgs Couplings and Precision Electroweak Data, JHEP 01 (2013) 139 [arXiv: 1209.6382] [INSPIRE].

[20] J.A. Aguilar-Saavedra, R. Benbrik, S. Heinemeyer and M. Pérez-Victoria, Handbook of vectorlike quarks: Mixing and single production, Phys. Rev. D 88 (2013) 094010 [arXiv: 1306.0572] [INSPIRE].

[21] C.-Y. Chen, S. Dawson and E. Furlan, Vectorlike fermions and Higgs effective field theory revisited, Phys. Rev. D 96 (2017) 015006 [arXiv:1703.06134] [INSPIRE].

[22] ATLAS and CMS collaborations, Measurements of the Higgs boson production and decay rates and constraints on its couplings from a combined ATLAS and CMS analysis of the LHC pp collision data at $\sqrt{s}=7$ and 8 TeV, JHEP 08 (2016) 045 [arXiv: 1606. 02266] [INSPIRE].

[23] K. Cheung, J.S. Lee and P.-Y. Tseng, New Emerging Results in Higgs Precision Analysis Updates 2018 after Establishment of Third-Generation Yukawa Couplings, JHEP 09 (2019) 098 [arXiv: 1810.02521] [INSPIRE].

[24] L. Lavoura and J.P. Silva, The Oblique corrections from vector-like singlet and doublet quarks, Phys. Rev. D 47 (1993) 2046 [InSPIRE].

[25] M. Carena, E. Ponton, J. Santiago and C.E.M. Wagner, Light Kaluza Klein States in Randall-Sundrum Models with Custodial SU(2), Nucl. Phys. B 759 (2006) 202 [hep-ph/0607106] [INSPIRE].

[26] C. Anastasiou, E. Furlan and J. Santiago, Realistic Composite Higgs Models, Phys. Rev. D 79 (2009) 075003 [arXiv:0901.2117] [INSPIRE].

[27] D. Silverman, The Full range of predictions for B physics from iso-singlet down quark mixing, Phys. Rev. D 58 (1998) 095006 [hep-ph/9806489] [INSPIRE].

[28] J.A. Aguilar-Saavedra, Effects of mixing with quark singlets, Phys. Rev. D 67 (2003) 035003 [Erratum ibid. D 69 (2004) 099901] [hep-ph/0210112] [INSPIRE].

[29] F.J. Gilman, Quark Mixing: The CKM Picture, Ann. Rev. Nucl. Part. Sci. 40 (1990) 213.

[30] W.-S. Hou, M. Kohda and F. Xu, Rates and asymmetries of $B \rightarrow \pi \ell^{+} \ell^{-}$decays, Phys. Rev. D 90 (2014) 013002 [arXiv:1403.7410] [InSPIRE].

[31] A.V. Rusov, Probing New Physics in $b \rightarrow d$ Transitions, arXiv:1911.12819 [InSPIRE].

[32] A.K. Alok, S. Banerjee, D. Kumar and S. Uma Sankar, Flavor signatures of isosinglet vector-like down quark model, Nucl. Phys. B 906 (2016) 321 [arXiv:1402.1023] [INSPIRE]. 
[33] LHCb collaboration, Search for lepton-universality violation in $B^{+} \rightarrow K^{+} \ell^{+} \ell^{-}$decays, Phys. Rev. Lett. 122 (2019) 191801 [arXiv: 1903.09252] [INSPIRE].

[34] BeLle collaboration, Test of lepton flavor universality in $B \rightarrow K^{*} \ell^{+} \ell^{-}$decays at Belle, arXiv: 1904.02440 [INSPIRE].

[35] LHCb collaboration, First measurement of the differential branching fraction and $C P$ asymmetry of the $B^{ \pm} \rightarrow \pi^{ \pm} \mu^{+} \mu^{-}$decay, JHEP 10 (2015) 034 [arXiv:1509.00414] [INSPIRE].

[36] ATLAS collaboration, Combination of the searches for pair-produced vector-like partners of the third-generation quarks at $\sqrt{s}=13 \mathrm{TeV}$ with the ATLAS detector, Phys. Rev. Lett. 121 (2018) 211801 [arXiv: 1808.02343] [INSPIRE].

[37] CMS collaboration, Search for vector-like quarks in events with two oppositely charged leptons and jets in proton-proton collisions at $\sqrt{s}=13$ TeV, Eur. Phys. J. C 79 (2019) 364 [arXiv: 1812.09768] [INSPIRE].

[38] CMS collaboration, Search for pair production of vectorlike quarks in the fully hadronic final state, Phys. Rev. D 100 (2019) 072001 [arXiv:1906.11903] [INSPIRE].

[39] ATLAS collaboration, Search for single production of a vector-like B quark decaying into a bottom quark and a Higgs boson which decays into a pair of photons,

ATLAS-CONF-2018-024 (2018).

[40] CMS collaboration, Search for pair production of vector-like quarks in the $b W \overline{\mathrm{b}} W$ channel from proton-proton collisions at $\sqrt{s}=13 \mathrm{TeV}$, Phys. Lett. B 779 (2018) 82 [arXiv: 1710.01539] [INSPIRE].

[41] ATLAS collaboration, Search for single production of vector-like quarks decaying into Wb in pp collisions at $\sqrt{s}=13 \mathrm{TeV}$ with the ATLAS detector, JHEP 05 (2019) 164 [arXiv: 1812.07343] [INSPIRE].

[42] ATLAS collaboration, Search for pair production of heavy vector-like quarks decaying into hadronic final states in pp collisions at $\sqrt{s}=13 \mathrm{TeV}$ with the ATLAS detector, Phys. Rev. D 98 (2018) 092005 [arXiv: 1808.01771] [INSPIRE].

[43] CMS collaboration, Search for vector-like $T$ and $B$ quark pairs in final states with leptons at $\sqrt{s}=13 \mathrm{TeV}$, JHEP 08 (2018) 177 [arXiv: 1805. 04758] [INSPIRE].

[44] ATLAS collaboration, Search for pair- and single-production of vector-like quarks in final states with at least one $Z$ boson decaying into a pair of electrons or muons in pp collision data collected with the ATLAS detector at $\sqrt{s}=13$ TeV, Phys. Rev. D 98 (2018) 112010 [arXiv: 1806.10555] [INSPIRE].

[45] CMS collaboration, Search for electroweak production of a vector-like T quark using fully hadronic final states, JHEP 01 (2020) 036 [arXiv: 1909.04721] [INSPIRE].

[46] L.-L. Chau and W.-Y. Keung, Comments on the Parametrization of the Kobayashi-Maskawa Matrix, Phys. Rev. Lett. 53 (1984) 1802 [InSPIRE].

[47] J. McDonald, CP violation for electroweak baryogenesis from mixing of Standard Model and heavy vector quarks, Phys. Rev. D 53 (1996) 645 [INSPIRE].

[48] G.C. Branco, D. Delepine, D. Emmanuel-Costa and F.R. Gonzalez, Electroweak baryogenesis in the presence of an isosinglet quark, Phys. Lett. B 442 (1998) 229 [hep-ph/9805302] [INSPIRE]. 